\title{
ANÁLISIS INTEGRAL DE LOS FLUJOS COMERCIALES ENTRE EL PERÚ Y CHINA CON MIRAS A LA FIRMA DE UN ACUERDO DE ALCANCE PARCIAL*
}

\author{
Carlos Gallardo Torres \\ UNIVERSIDAD DEL PACÍFICO \\ cgallardo18@yahoo.es
}

\begin{abstract}
Resumen
La integración comercial y el reconocimiento de su papel como impulsora del desarrollo económico han sido parte de las prioridades del Perú a partir de su apertura económica. Siguiendo este lineamiento, resulta de fácil comprensión la decisión peruana de iniciar negociaciones de acuerdos comerciales preferenciales con sus principales socios. La presente investigación parte del escenario de una potencial negociación comercial entre el Perú y la República Popular de China y centra su análisis en una metodología que integra el cálculo de indicadores comerciales y la elaboración de matrices de clasificación de subpartidas y de identificación de potencialidades comerciales. El análisis de los resultados permitirá identificar los productos que están aprovechando las oportunidades de la relación comercial actual entre el Perú y China, así como los productos que potencialmente podrán beneficiarse de la apertura de mercados una vez que se establezca un acuerdo comercial entre ambos países. Asimismo, la investigación permitirá la elaboración de un ranking de subpartidas que reconozca aquellos sectores y productos en los que el Perú debería focalizar las potenciales negociaciones comerciales con este país.
\end{abstract}

Palabras clave: indicadores comerciales, negociación comercial, China, Perú.

\begin{abstract}
Trade and its role in economic development have been amongst Peru's priorities since the country's economic opening. Along this line, it is easy to understand Peru's decision to start negotiations to establish trade preference agreements with its main trading partners. This research starts by presenting the scenario for potential trade negotiations between Peru and the People's Republic of China. The analysis is based on a methodology integrating calculations of trade indicators and the design of tariff item classification and potential identification matrices. Our outcomes' analysis allows identifying those goods that are actually taking advantage of present trade relations between Peru and China, and other goods that might take further advantage of more open markets once a trade agreement is reached between the two nations. In addition, our research allows preparing a ranking of product classifications taking account of those industries and products where Peru should focus during trade talks with China.
\end{abstract}

Key words: trade indicators, trade negotiations, China, Peru.

* Trabajo premiado en el II Concurso de Investigación Juan Abugattás Abugattás, convocado en el 2005 por la Red Peruana para Estudios del Asia Pacífico (Redap). 


\section{Introducción}

Una tendencia claramente identificable ha convertido a la integración comercial en uno de los impulsores principales del desarrollo económico. El Perú no ha permanecido ajeno a este proceso, lo que se refleja en la reestructuración de su política de estabilización económica y de apertura comercial desde inicios de la década pasada (Aráoz y Casas, 1999). Como medio para alcanzar este objetivo, el Perú ha iniciado negociaciones comerciales con Estados Unidos y el Reino de Tailandia, y se espera que en el futuro estas se extiendan a Chile, Singapur y la Unión Europea $^{1}$, lo que permitirá, mediante la reducción de las barreras arancelarias y no arancelarias, el acceso a mercados más grandes y dinámicos.

Sin embargo, en el caso de algunos países, resulta útil establecer un mayor acercamiento comercial entre las economías mediante la firma de un acuerdo de carácter parcial, como paso previo a la integración comercial vía la firma de un acuerdo comercial preferencial (ACP). En este contexto, uno de los acuerdos comerciales de mayor envergadura al que el Perú podría aspirar sería aquel que se negocie con el mayor mercado asiático y una de las economías de mayor crecimiento en el mundo: la República Popular de China.

El presente estudio se sustenta en tres aspectos principales: a) la relevancia de China como actor en el comercio internacional y sus amplias perspectivas de de-

1. En el caso de las actuales negociaciones con Estados Unidos, el Perú comparte una posición conjunta con Ecuador y Colombia. Con la Unión Europea, la negociación será en conjunto con la Comunidad Andina de Naciones (CAN). sarrollo como mercado, b) la importancia para China de promover la facilitación del comercio en el marco de sus compromisos como economía miembro del APEC y como medio para alcanzar las metas planteadas en Bogor ${ }^{2}$ (IAP Study Group, 1997) y c) el gran interés chino por lograr una mayor integración al sistema de comercio internacional ${ }^{3}$.

Asimismo, otros factores hacen de la economía china un socio comercial deseable. En el ámbito mundial, el surgimiento y desarrollo de China como gran protagonista del comercio -resultado de sucesivas oleadas de reformas modernizadoras y de la apertura de su economía como parte de los compromisos asumidos al integrarse a la OMC en el 2001- es considerado uno de los factores determinantes

2. Dentro del marco de su apertura comercial y del cumplimiento de su compromiso con las metas de Bogor, en el ámbito del APEC, China ha mantenido la tendencia a la baja de sus aranceles, desde un promedio de 34,5\% en 1994 hasta $10,4 \%$ en el 2004 . Lo mismo ha sucedido con sus barreras no arancelarias (principalmente referidas a aspectos sanitarios y fitosanitarios), que ha buscado disminuir mediante la firma de convenios bilaterales o multilaterales.

3. Interés que se expresa partiendo de sus reformas estructurales en 1978, pasando por su incorporación como miembro de la Organización Mundial del Comercio (OMC) en el año 2001 y ratificándolo mediante sus negociaciones bilaterales alrededor del mundo (Delage, 2004; Guardia, 2004), como los que en la actualidad mantiene o ha logrado concretar con: los diez países de la Asociación de Naciones del Sudeste Asiático (Asean), Australia, Bolivia, Chile, Corea, el bloque Arabia Saudí, Bahréin, Kuwait, Omán, Qatar y Emiratos Árabes Unidos (Consejo de Cooperación de los Estados Árabes del Golfo-GCC), Japón, Rusia, Nueva Zelanda, Pakistán, Tailandia e Islandia (Dirección General de Relaciones Económicas Internacionales de Chile, www.direcon.cl). 
de las tendencias actuales del comercio mundial de mercancías ${ }^{4}$ (OMC, 2004). De igual manera, en el ámbito latinoamericano China se ha mantenido en los últimos años como el principal destino de las exportaciones de la región. En el año 2003, las exportaciones latinoamericanas a ese país se incrementaron en 9200 millones de dólares (OMC, 2004). Finalmente, en lo referido a las relaciones comerciales bilaterales, China ha pasado a convertirse en el segundo socio comercial del Perú en el año $2004^{5}$ y en uno de los principales motores de su crecimiento exportador.

De esta forma, partiendo de la importancia de la economía china en sus diferentes ámbitos, así como la del comercio bilateral entre el Perú y China, y dentro del contexto de una potencial negociación comercial entre ambos países, la presente investigación analiza de manera integral los flujos comerciales mediante el uso de indicadores y la elaboración de una matriz de clasificación y dos matrices de potencialidades comerciales.

El trabajo se divide en tres secciones: en la primera se realiza un estudio des-

4. En el aspecto comercial, la economía china se convirtió en el año 2003 en el cuarto exportador más grande del mundo, con un total de 438 mil millones de dólares, luego de Alemania, Estados Unidos y Japón. Asimismo, se convirtió en el tercer importador más grande del mundo, luego de Estados Unidos y Alemania, con un monto de 413 mil millones de dólares. Finalmente, se debe rescatar que China es el primer destino de la inversión extranjera directa (IED) en el mundo, ya que captó alrededor de 54 mil millones de dólares en el 2003, más del 50\% destinado a la región asiática.

5. El crecimiento de las exportaciones peruanas a ese destino (82\%) entre el 2003 y el 2004 desplazó a Gran Bretaña del segundo lugar como socio comercial (Cómex Perú, 2005). criptivo de los flujos comerciales entre el Perú y China a lo largo del periodo 19932003. En la segunda sección se analiza tanto indicadores comerciales como matrices comerciales de clasificación y de potencialidad, lo que culmina con la integración de ambas metodologías y permite, en la tercera y última sección, llegar a conclusiones sobre la priorización de las subpartidas y de los sectores productivos con miras a una potencial negociación comercial con China.

\section{Objetivos, alcances e hipótesis}

A través del desarrollo de una metodología que integra y permite la interpretación conjunta de algunos indicadores de flujos comerciales y los resultados de la construcción de una matriz de potencialidades de comercio, la presente investigación intenta contribuir a la presentación de una propuesta peruana que establezca una priorización de subpartidas frente al escenario de una potencial negociación comercial con China.

Los indicadores de flujos comerciales que se calculan en este trabajo son los siguientes:

- Ventaja comparativa revelada (VCR) para las exportaciones peruanas totales.

- Desventaja comparativa revelada (DCR) para las importaciones Chinas.

- Complementariedad (IC) de los flujos comerciales entre el Perú y China.

- Especialización (IE) de los productos peruanos en el mercado chino.

- Cuota china de importaciones del mercado mundial $(\mathrm{CN})$. 
Por su parte, la construcción de una matriz de clasificación y dos de potencialidades comerciales permitirá identificar aquellas subpartidas que en la actualidad aprovechan las oportunidades de la relación comercial entre el Perú y China, o que podrían hacerlo si esta se intensificara en un futuro vía la firma de un acuerdo de comercio preferencial.

La limitación más importante de este trabajo es la escasez de información sobre China, lo que ocasiona que este estudio no considere el cálculo de indicadores ni la construcción de las matrices comerciales para este país, con lo que se habría logrado un análisis comparativo más completo y la obtención de mejores conclusiones.

Un análisis más profundo debería tomar en cuenta, para cada subpartida, un estudio de las ventajas y desventajas en dicho mercado; en tal sentido, es de suma importancia conocer las trabas existentes al libre comercio, ya sean de carácter arancelario o no arancelario, así como los principales competidores en el mercado.

El desarrollo de una metodología integral y su aplicación a los flujos comerciales bilaterales entre el Perú y China permitirá la elaboración de un ranking de subpartidas que reconozca aquellos sectores y productos en los que el Perú debería focalizar las potenciales negociaciones comerciales con este país.

Especificando, el presente trabajo contempla la siguiente hipótesis principal:

La metodología aplicada recomendará al Perú la focalización de su propuesta de negociación en aquellas subpartidas que obtengan índices de
VCR e IE mayor de 1 y que a su vez se encuentren dentro del cuadrante superior derecho de las matrices de clasificación y potencialidad, y en las cuales China tenga una mayor cuota de mercado. Las subpartidas que cumplirán dichos requerimientos serán principalmente las pertenecientes a los subsectores pesquero y minero, dentro del sector tradicional, y a los subsectores pesca y textil, dentro del sector no tradicional.

A la vez, plantea las siguientes hipótesis secundarias:

- El resultado de la aplicación de los indicadores comerciales indicará que el Perú posee ventajas comparativas en la mayoría de productos exportados a China y que se encuentra especializado en el mercado chino, principalmente en las subpartidas pertenecientes a los subsectores pesquero y minero, dentro del sector tradicional, y a los subsectores pesca y textil, dentro del sector no tradicional. Además, ambas economías resultan complementarias en lo que respecta a sus flujos comerciales.

- A través del uso de la matriz de clasificación, la mayor parte de las subpartidas se ubicarán dentro del cuadrante en el que la tasa de crecimiento de las exportaciones peruanas a China y las importaciones totales de esta última son mayores a sus respectivos promedios. Por su parte, la matriz de potencialidades señalará una concentración de las subpartidas dentro del escenario en el que las tasas de crecimiento de las exportaciones peruanas y de las importaciones chinas son mayores a sus respectivos promedios, hacia y 
desde el resto del mundo, respectivamente.

\section{Análisis integral del flujo comercial entre el Perú y China}

El alto crecimiento exportador que el Perú ha experimentado en los últimos años le permitió alcanzar, en el 2004, un récord de casi 12,5 mil millones de dólares, de los cuales el 33,37\% se dirigió a América del Norte; el 24,33\%, a la Unión Europea; y el $18,1 \%$, a los países de Asia; mientras que la Comunidad Andina (CAN) representó sólo el 6\%; Chile, el 5,14\%; y el Mercosur, el 3,22\% ${ }^{6}$.

Entre los países asiáticos, China se ha consolidado como actor principal del comercio peruano, no sólo por ser destino del 55\% de las exportaciones peruanas a ese continente -para el año 2004 -, sino también por haberse consolidado como el segundo destino de las exportaciones totales del Perú en ese mismo año, sólo después de Estados Unidos. Por estos motivos, una negociación que mejore las relaciones comerciales bilaterales podría brindar grandes oportunidades para ambos países en lo que se refiere a diversificación e intensificación del volumen actual de exportaciones.

Como se observa en el gráfico 1, tanto las importaciones desde China como las exportaciones hacia ese país muestran una tendencia creciente y relativamente estable. Con excepción del año 1999, el intercambio comercial bilateral ha sido superavitario para el Perú. A partir de 1993, las importaciones peruanas desde China registran un crecimiento total de $749 \%$, pasando de un poco menos de 90,5 millones de dólares a casi 767,5 millones de dólares en el 2004. De manera análoga, las exportaciones peruanas a China, a pesar de mostrar altibajos entre 1993 y 2004, presentan un crecimiento de $777 \%$ y alcanzaron los 1235 millones de dólares en el último año.

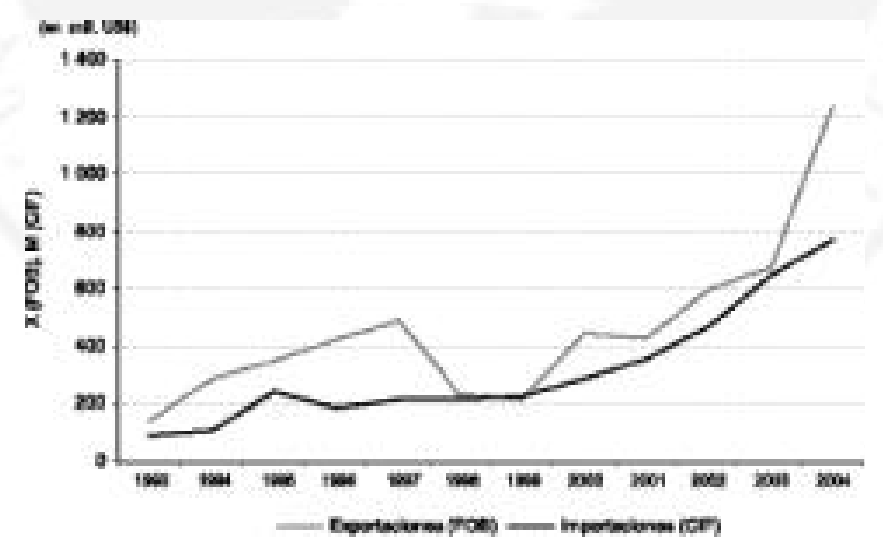

Gráfico 1

Intercambio comercial Perú-China 1993-2004

6. Fuente: Comisión para la Promoción de Exportaciones (Prómpex). 
Las exportaciones peruanas a China, a pesar de su alto crecimiento, presentan una muy baja diversificación: del total de productos del universo arancelario ${ }^{7}$, en el año 2004 sólo se exportaron 240 productos, de los cuales los 10 primeros representaron el 95,98\% del total (ver cuadro 1). Los mayores montos de exportación corresponden al sector minero, seguido del sector pesquero y del siderúrgico y metalúrgico (ver cuadro 2).
Por otro lado, las importaciones desde China presentan una mayor diversificación: del total importado en el año 2004 (767 millones de dólares), los 20 productos principales apenas representan el $33,27 \%$ del monto total (ver cuadro 3 ). Los mayores montos de importación se concentran en los sectores metal-mecánico, químico y textil, con el $25,24 \%, 7,36 \%$ y $4,45 \%$ de las importaciones totales, respectivamente (ver cuadro 4 ).

Cuadro 1. Principales productos exportados a China (2004)

\begin{tabular}{|c|c|c|c|c|c|}
\hline Ord. & Producto & Descripción & FOB US\$ & $\%$ & Sector \\
\hline 1 & 2301201010 & $\begin{array}{l}\text { Harina de pescado sin desgrasar, } \\
\text { impropia para la alimentación humana, } \\
\text { con contenido de grasa }>2 \% \text { en peso. }\end{array}$ & 425395082 & 34,44 & Pesca \\
\hline 2 & 2603000000 & Minerales de cobre y sus concentrados. & 404805998 & 32,77 & Minería \\
\hline 3 & 2607000000 & Minerales de plomo y sus concentrados. & 103982229 & 8,42 & Minería \\
\hline 4 & 7403110000 & $\begin{array}{l}\text { Cátodos y secciones de cátodos de cobre } \\
\text { refinado. }\end{array}$ & 78547046 & 6,36 & $\begin{array}{l}\text { Siderúrgico y } \\
\text { metalúrgico }\end{array}$ \\
\hline 5 & 2601120000 & $\begin{array}{l}\text { Minerales de hierro y sus concentrados, } \\
\text { aglomerados. }\end{array}$ & 52399668 & 4,24 & Minería \\
\hline 6 & 2613900000 & $\begin{array}{l}\text { Minerales de molibdeno y sus concentra- } \\
\text { dos, sin tostar. }\end{array}$ & 40432882 & 3,27 & Minería \\
\hline 7 & 2601110000 & $\begin{array}{l}\text { Minerales de hierro y sus concentrados, } \\
\text { sin aglomerar. }\end{array}$ & 38477928 & 3,11 & Minería \\
\hline 8 & 7402001000 & Cobre «blister» sin refinar. & 15568922 & 1,26 & Minería \\
\hline 9 & 1605909000 & $\begin{array}{l}\text { Demás moluscos e invertebrados } \\
\text { acuáticos, preparados o conservados. }\end{array}$ & 14623246 & 1,18 & Pesca \\
\hline 10 & 0307490000 & $\begin{array}{l}\text { Demás jibias, globitos, calamares y } \\
\text { potas, congeladas, secas, saladas o en } \\
\text { salmuera. }\end{array}$ & 11438016 & $\begin{array}{r}0,93 \\
95,98\end{array}$ & Pesca \\
\hline
\end{tabular}

Fuente: ADEX Data Trade.

Elaboración propia.

7. Según la clasificación a 10 dígitos del Sistema Armonizado de Designación y Codificación de Mercancías. 
Cuadro 2. Principales sectores de exportación peruana a China (2004)

\begin{tabular}{lrr}
\hline \multicolumn{1}{c}{ Sectores } & Valor FOB US $\$$ & $\%$ \\
\hline Minería & 744545016,22 & $60,25 \%$ \\
Pesquero & 426555397,12 & $34,52 \%$ \\
Pesca & 28876793,27 & $2,34 \%$ \\
Textil & 12513376,52 & $1,01 \%$ \\
Varios & 8411356,28 & $0,68 \%$ \\
Siderúrgico y metalúrgico & 5653333,14 & $0,46 \%$ \\
Químico & 4884995,73 & $0,40 \%$ \\
Agrario & 2290198,12 & $0,19 \%$ \\
Agropecuario y agroindustrial & 1380111,88 & $0,11 \%$ \\
Metal-mecánico & 428854,00 & $0,03 \%$ \\
Petróleo & 83465,06 & $0,01 \%$ \\
Minería no metálica & 66491,70 & $0,01 \%$ \\
Prendas de vestir & 7147,88 & $0,00 \%$ \\
Total general & 1235696536,92 & $100,00 \%$ \\
\hline
\end{tabular}

Fuente: PrompexStat.

Elaboración propia.

Cuadro 4. Principales sectores de importación peruana desde China (2004)

\begin{tabular}{lrr}
\hline \multicolumn{1}{c}{ Sectores } & Valor FOB USD. & $\%$ \\
\hline Metal-mecánico & 387376367 & $25,24 \%$ \\
Químico & 112925804 & $7,36 \%$ \\
Textil & 68329700 & $4,45 \%$ \\
Minería no metálica & 25252037 & $1,65 \%$ \\
Siderúrgico y metalúrgico & 19872233 & $1,29 \%$ \\
Prendas de vestir & 7726868 & $0,50 \%$ \\
Agropecuario y agroindustrial & 4783270 & $0,31 \%$ \\
Agrario & 406760 & $0,03 \%$ \\
Pesca & 215342 & $0,01 \%$ \\
Minería & 111491 & $0,01 \%$ \\
Petróleo & 55 & $0,00 \%$ \\
Varios & 907947800 & $59,15 \%$ \\
Total general & 1534947727 & $100,00 \%$ \\
\hline
\end{tabular}

Fuente: ADEX Data Trade.

Elaboración propia. 
Cuadro 3. Principales productos importados desde China (2004)

\begin{tabular}{|c|c|c|c|c|c|}
\hline Ord & Partida & Descripción & CIF US\$ & $\%$ & Sector \\
\hline 1 & 8521900000 & $\begin{array}{l}\text { Los demás aparatos de grabación y repro- } \\
\text { ducción de imagen y sonido. }\end{array}$ & 35137639 & 4,58 & Varios \\
\hline 2 & 852520110 & Teléfonos. & 26450154 & 3,45 & Metal-mecánico \\
\hline 3 & 8471700000 & Unidades de memoria. & 17265752 & 2,25 & Metal-mecánico \\
\hline 4 & 8527310000 & $\begin{array}{l}\text { Demás aparatos receptores de radiodifu- } \\
\text { sión combinados con grabador o reproduc- } \\
\text { tor de sonido. }\end{array}$ & 17051748 & 2,22 & Metal-mecánico \\
\hline 5 & 8471500000 & $\begin{array}{l}\text { Unidades de proceso digitales, excepto } \\
\text { las de las subpartidas } n .^{\circ} 8471.41 .00 \text { y } \\
8471.49 .00 \ldots\end{array}$ & 16717224 & 2,18 & Metal-mecánico \\
\hline 6 & 8711200000 & $\begin{array}{l}\text { Motocicletas y velocípedos con motor de } \\
\text { émbolo alternativo, } 50 \mathrm{cc}<\text { cil. }<250 \mathrm{cc} \text {. }\end{array}$ & 15910803 & 2,07 & Metal-mecánico \\
\hline 7 & 8473300000 & $\begin{array}{l}\text { Partes y accesorios de máquinas de la } \\
\text { partida } n .^{\circ} 84.71 \text {. }\end{array}$ & 14594735 & 1,9 & Metal-mecánico \\
\hline 8 & 6402990000 & Los demás calzados. & 14418490 & 1,88 & Varios \\
\hline 9 & 8528129000 & Los demás aparatos receptores de TV. & 11067847 & 1,44 & Metal-mecánico \\
\hline 10 & 8471300000 & $\begin{array}{l}\text { Máquinas automáticas para tratamiento/ } \\
\text { procesamiento de datos, digitales, portáti- } \\
\text { les peso }<=10 \mathrm{~kg} \text {. }\end{array}$ & 10948824 & 1,43 & Metal-mecánico \\
\hline 11 & 6006320000 & Teñidos. & 10337839 & 1,35 & Textil \\
\hline 12 & 2835310000 & Trifosfato de sodio (tripolifosfato de sodio). & 9812180 & 1,28 & Químico \\
\hline 13 & 5211420000 & $\begin{array}{l}\text { Tejidos de algodón con hilados de colores } \\
\text { de gramaje }>200 \mathrm{~g} / \mathrm{m}^{2} \text { de mezclilla («de- } \\
\text { nim»). }\end{array}$ & 7725781 & 1,01 & Textil \\
\hline 14 & 8517500000 & $\begin{array}{l}\text { Demás aparatos de telecomunicación por } \\
\text { corriente portadora o telecomunicación di- } \\
\text { gital. }\end{array}$ & 7664668 & 1,00 & Metal-mecánico \\
\hline 15 & 8471609000 & $\begin{array}{l}\text { Las demás unidades de entrada o salida, } \\
\text { aunque incluyan unidades de memoria en } \\
\text { envoltura. }\end{array}$ & 7526759 & 0,98 & Metal-mecánico \\
\hline 16 & 4011200000 & $\begin{array}{l}\text { Neumáticos nuevos de caucho del tipo de } \\
\text { los utilizados en autobuses o camiones. }\end{array}$ & 6995890 & 0,91 & Químico \\
\hline 17 & 3926909090 & $\begin{array}{l}\text { Demás manufacturas de plástico y manu- } \\
\text { facturas de las demás materias. De parti- } \\
\text { da } 39.01 \text { a } 39.14 \text {. }\end{array}$ & 6576942 & 0,86 & Químico \\
\hline 18 & 8471601000 & Impresoras. & 6361295 & 0,83 & Metal-mecánico \\
\hline 19 & 9503900000 & Los demás juguetes para entretenimiento. & 6353675 & 0,83 & Varios \\
\hline 20 & 8525201900 & $\begin{array}{l}\text { Los demás aparatos emisores con apara- } \\
\text { to receptor incorporado. }\end{array}$ & 6260944 & 0,82 & Metal-mecánico \\
\hline
\end{tabular}

Fuente: ADEX Data Trade.

Elaboración propia. 


\section{Metodología}

La metodología aplicada en la presente investigación consta de dos tipos de abordaje. El primero de ellos consiste en el cálculo de indicadores de comercio internacional, los que permitirán evaluar la tendencia de los flujos comerciales entre el Perú y China con la intención de reconocer productos que presenten ventajas comparativas reveladas o en los cuales el país posea algún grado de especialización. El segundo abordaje consiste en la construcción de tres matrices: una de clasificación de productos y dos de potencialidades comerciales, con las que se podrán identificar los productos que están aprovechando las oportunidades de la relación comercial actual entre el Perú y China, así como los productos que potencialmente podrán aprovechar la apertura de mercados una vez que se establezca un acuerdo comercial entre dichos países.

Finalmente, se realiza un análisis integral que examina transversalmente ambos abordajes, de modo que puedan formularse algunas conclusiones respecto de la priorización de subpartidas ante la firma de un acuerdo de alcance parcial entre el Perú y China.

\subsection{Herramientas de comercio internacional}

Con el propósito de analizar los flujos comerciales entre el Perú y China se calculan algunos indicadores comerciales relevantes ${ }^{8}$ ya mencionados, los índices de: ventajas comparativas reveladas (VCR), desventajas comparativas revela-

8. Para mayores detalles sobre el cálculo de los índices, véase el anexo. das (DCR), especialización (IE) y complementariedad $^{9}$.

\subsubsection{Indicador de ventajas comparativas reveladas (VCR) para el Perú}

Analizado desde la perspectiva ricardiana, el índice de VCR otorga señales de que el costo de oportunidad de la producción de la subpartida analizada, respecto de otras subpartidas, es menor para el Perú que para sus competidores en el extranjero. De esta forma, aquellas subpartidas con índice de VCR mayor de 1 serán prioritarias, pues su producción podría ser considerada como eficiente para el país.

Del conjunto de subpartidas exportadas por el Perú en el 2003 (a 6 dígitos del sistema armonizado), 443 presentaron un índice de VCR mayor de 1 (ver cuadro 5). Si el total de subpartidas con VCR mayor de 1 se observa como porcentaje de exportación por sectores, el 92,6\% del volumen de las exportaciones peruanas presenta ventajas comparativas. Los sectores con mayores resultados son: pesquero $(100 \%)$, pesca $(99,7 \%)$, agrario $(97,6 \%)$ y siderúrgico y metalúrgico $(95,8 \%)$. Los de menor participación son los derivados del petróleo $(0,1 \%)$ y el metal-mecánico $(20,6 \%)$.

\subsubsection{Indicador de desventajas comparativas reveladas (DCR) para China}

En forma análoga a la interpretación del índice de VCR, si una subpartida presenta

9. Por limitaciones de disponibilidad de datos, los índices sólo podrán ser calculados para el año 2003, lo que limita el análisis de sus resultados. 
Cuadro 5. Número y participación de las subpartidas con VCR $>1$ en las exportaciones peruanas (2003)

\begin{tabular}{lcrrr}
\hline \multicolumn{1}{c}{ Sector } & $\begin{array}{c}\text { N. }{ }^{\circ} \text { Subpartidas } \\
\text { con VCR }>\mathbf{1}\end{array}$ & $\begin{array}{c}\text { X Peruanas (US\$) } \\
\text { con VCR }>\mathbf{1}\end{array}$ & $\begin{array}{c}\text { X Perú totales } \\
\text { (US } \$ \text { ) }\end{array}$ & $\begin{array}{r}\text { \% respecto } \\
\text { de X total }\end{array}$ \\
\hline Agrario & 15 & 217257269 & 222661722 & $97,6 \%$ \\
Agropecuario y agroindustrial & 84 & 570027306 & 616732239 & $92,4 \%$ \\
Metal-mecánico & 19 & 20893639 & 101184794 & $20,6 \%$ \\
Minería & 21 & 4236734694 & 4239266527 & $99,9 \%$ \\
Minería no metálica & 23 & 54160184 & 72288015 & $74,9 \%$ \\
Pesca & 31 & 944665575 & 947732896 & $99,7 \%$ \\
Pesquero & 2 & 79079836 & 79079836 & $100,0 \%$ \\
Petróleo & 3 & 233792 & 266857711 & $0,1 \%$ \\
Prendas de vestir & 37 & 602144677 & 647145156 & $93,0 \%$ \\
Químico & 53 & 629961718 & 713384454 & $88,3 \%$ \\
Siderúrgico y metalúrgico & 32 & 367717743 & 383662143 & $95,8 \%$ \\
Textil & 77 & 153797464 & 173847701 & $88,5 \%$ \\
Varios & 46 & 224137385 & 288738923 & $77,6 \%$ \\
Total general & 443 & 8100811282 & 8752582117 & $92,6 \%$ \\
\hline
\end{tabular}

Fuente: Ministerio de Comercio Exterior y Turismo.

Elaboración propia.

Cuadro 6. Número y participación de las subpartidas con DCR $>1$ en las importaciones chinas (2003)

\begin{tabular}{|c|c|c|c|c|}
\hline Sector & $\begin{array}{c}N .{ }^{\circ} \text { Subpartidas } \\
\text { con } \mathrm{DCR}>1\end{array}$ & $\begin{array}{c}\text { M Chinas (US\$) } \\
\text { con DCR >1 }\end{array}$ & $\begin{array}{c}\text { M China totales } \\
\text { (US\$) }\end{array}$ & $\begin{array}{c}\% \text { respecto } \\
\text { de } X \text { total }\end{array}$ \\
\hline Agrario & 11 & 2126066 & 2323434 & $91,5 \%$ \\
\hline Agropecuario y agroindustrial & 56 & 10406138 & 12734844 & $81,7 \%$ \\
\hline Metal-mecánico & 442 & 118662403 & 155208400 & $76,5 \%$ \\
\hline Minería & 22 & 11169138 & 11364787 & $98,3 \%$ \\
\hline Minería no metálica & 39 & 2888805 & 4337108 & $66,6 \%$ \\
\hline Pesca & 20 & 2001877 & 2354749 & $85,0 \%$ \\
\hline Pesquero & 0 & - & 8694 & $0,0 \%$ \\
\hline Petróleo & 5 & 22216152 & 22284496 & $99,7 \%$ \\
\hline Prendas de vestir & 1 & 199658 & 1185130 & $16,8 \%$ \\
\hline Químico & 314 & 48194570 & 58778836 & $82,0 \%$ \\
\hline Siderúrgico y metalúrgico & 155 & 28419978 & 31997863 & $88,8 \%$ \\
\hline Textil & 262 & 13186591 & 14675412 & $89,9 \%$ \\
\hline Varios & 150 & 30753915 & 37379412 & $82,3 \%$ \\
\hline Total general & 1477 & 290225291 & 354633165 & $81,8 \%$ \\
\hline
\end{tabular}

Fuente: Ministerio de Comercio Exterior y Turismo.

Elaboración propia. 
un índice de DCR mayor de 1, el país posee una desventaja en el comercio internacional de ese bien, por lo que tenderá a ser un importador del mismo. En este caso, China presenta un índice DCR mayor de 1 en 1477 partidas de las 4574 partidas que importa en total (ver cuadro 6). La desventaja se presenta, principalmente, en los sectores metal-mecánico (442), químico (314) y textil (155). Cabe precisar que los últimos dos sectores son justamente aquellos donde se encuentran las subpartidas con los mayores resultados del índice de VCR calculado para el Perú.

Asimismo, las subpartidas que poseen un índice DCR mayor de 1 representan el $81,8 \%$ del volumen total de importaciones chinas y corresponden, principalmente, a los sectores petrolero $(99,7 \%)$, minero $(98,3 \%)$ y agrario $(91,5 \%)$. Cabe señalar que los últimos dos sectores son justamente aquellos donde también se encuentran los mayores porcentajes de subpartidas con índice de VCR mayor de 1 calculado para el Perú.

\subsubsection{Indicador de especialización (IE) para el Perú en el mercado chino}

El índice de especialización busca identificar los mercados en los que el Perú presenta tal grado de participación que sus exportaciones se ven especializadas en aquel mercado. Los resultados indican que 443 subpartidas del total exportado a China presentan un resultado mayor de 1 en este índice (ver cuadro 7). Estas partidas suman más de 667 millones de dólares en exportación, lo que representa el $98,7 \%$ del total exportado a ese país. Cabe resaltar que en la mayoría de los sectores, las exportaciones peruanas se encuentran especializadas en el mercado de exportación chino.

\section{Cuadro 7. Número y participación de las subpartidas con IE >1} en las exportaciones peruanas a China (2003)

\begin{tabular}{lrrrr}
\hline \multicolumn{1}{c}{ Sector } & $\begin{array}{c}\text { N. }{ }^{\circ} \text { subpartidas } \\
\text { con IE > } \mathbf{1}\end{array}$ & $\begin{array}{r}\text { X Perú a China } \\
\text { con IE > 1 (US\$) }\end{array}$ & $\begin{array}{r}\text { X Perú a China } \\
\text { totales (US\$) }\end{array}$ & $\begin{array}{r}\text { \% respecto de X } \\
\text { a China total }\end{array}$ \\
\hline Agrario & 15 & 248687 & 251122 & $99,0 \%$ \\
Agropecuario y agroindustrial & 84 & 720619 & 735278 & $98,0 \%$ \\
Metal-mecánico & 19 & 313767 & 539156 & $58,2 \%$ \\
Minería & 21 & 369320495 & 371003917 & $99,5 \%$ \\
Minería no metálica & 23 & - & 7938 & $0,0 \%$ \\
Pesca & 31 & 285662372 & 285688159 & $100,0 \%$ \\
Pesquero & 2 & 1061736 & 1061736 & $100,0 \%$ \\
Petróleo & 3 & - & & - \\
Prendas de vestir & 37 & 1500 & 2431 & $61,7 \%$ \\
Químico & 53 & 721516 & 1034753 & $69,7 \%$ \\
Siderúrgico y metalúrgico & 32 & 3947171 & 4079058 & $96,8 \%$ \\
Textil & 77 & 3602213 & 9849898 & $36,6 \%$ \\
Varios & 46 & 2291295 & 2718955 & $84,3 \%$ \\
Total general & 443 & 667891370 & 676972400 & $98,7 \%$ \\
\hline
\end{tabular}

Fuente: Ministerio de Comercio Exterior y Turismo.

Elaboración propia. 


\subsubsection{Indicador de complementariedad entre el Perú y China}

Como último paso, partiendo de los resultados encontrados para los índices de VCR del Perú y de DCR de China, el índice de complementariedad analiza la compatibilidad de la estructura de flujos comerciales entre ambos países para el año 2003. En este caso, la cifra de 1,52 revela que ambas economías son complementarias. Este resultado respalda la intuición de que, en gran parte de las subpartidas analizadas, altos índices de DCR para China corresponden a altos índices de VCR para el Perú. Asimismo, lleva a establecer el supuesto de que el Perú tiende a producir los bienes que China importa, y viceversa, por lo que un acuerdo comercial entre estos países podría resultar beneficioso para ambos.

\subsection{Análisis de las matrices comerciales}

\subsubsection{Matriz de clasificación}

Para la elaboración de esta matriz se consideró sólo aquellas subpartidas nacionales de las cuales se hubiesen exportado a China, en promedio, más de 10 mil dólares por año durante el periodo 1999-2003. Además, se tomó en cuenta únicamente las subpartidas que hubiesen sido exportadas al menos dos años. De esa forma, el número total de subpartidas resultantes fue de 65 .

Para elaborar el gráfico de los resultados se debe calcular lo siguiente: a) para el eje «x», la tasa de crecimiento de las subpartidas exportadas por el Perú a China; (b) para el eje «y», la tasa de crecimiento de las subpartidas importadas por China desde el mundo; c) para señalar el tamaño del mercado, las importaciones totales chinas del año 2003; y d) para establecer la separación de los cuadrantes, las tasas de crecimiento promedio de las exportaciones peruanas y de las importaciones chinas hacia y desde el mundo, respectivamente. Una vez calculados los valores y con la finalidad de lograr una mejor percepción de la matriz de clasificación, en el gráfico 2 se presentan sólo los 25 principales mercados chinos ${ }^{10}$.

Luego, considerando que las exportaciones peruanas al mundo han crecido $10 \%$ anual, en promedio, durante el periodo 1999-2003, que las importaciones chinas desde el mundo han crecido en $26 \%{ }^{11}$ y estableciendo un ranking según el tamaño de mercado, la clasificación de la totalidad de subpartidas (65) se presenta a continuación.

\section{a) Primer cuadrante: oportunidades aprovechadas}

Este primer cuadrante clasifica a aquellas subpartidas que el Perú exportó a China y que este país importó del mundo, y que en ambos casos crecieron por encima de sus respectivos promedios. Lo anterior significa que el Perú está aprovechando dichos mercados al exportar cada vez más frente a una demanda creciente. El número de subpartidas incluidas en el primer cuadrante es de 12, lo que en el 2003 equivalió a un valor de exportaciones perua-

10. Las subpartidas 260800 y 510710 no han sido incorporadas en el gráfico 2 con la finalidad de que la escala permita una mejor percepción del mismo.

11. Las tasas de crecimiento de las exportaciones e importaciones totales del Perú y China, respectivamente, se calcularon según información proporcionada por el Fondo Monetario Internacional. 


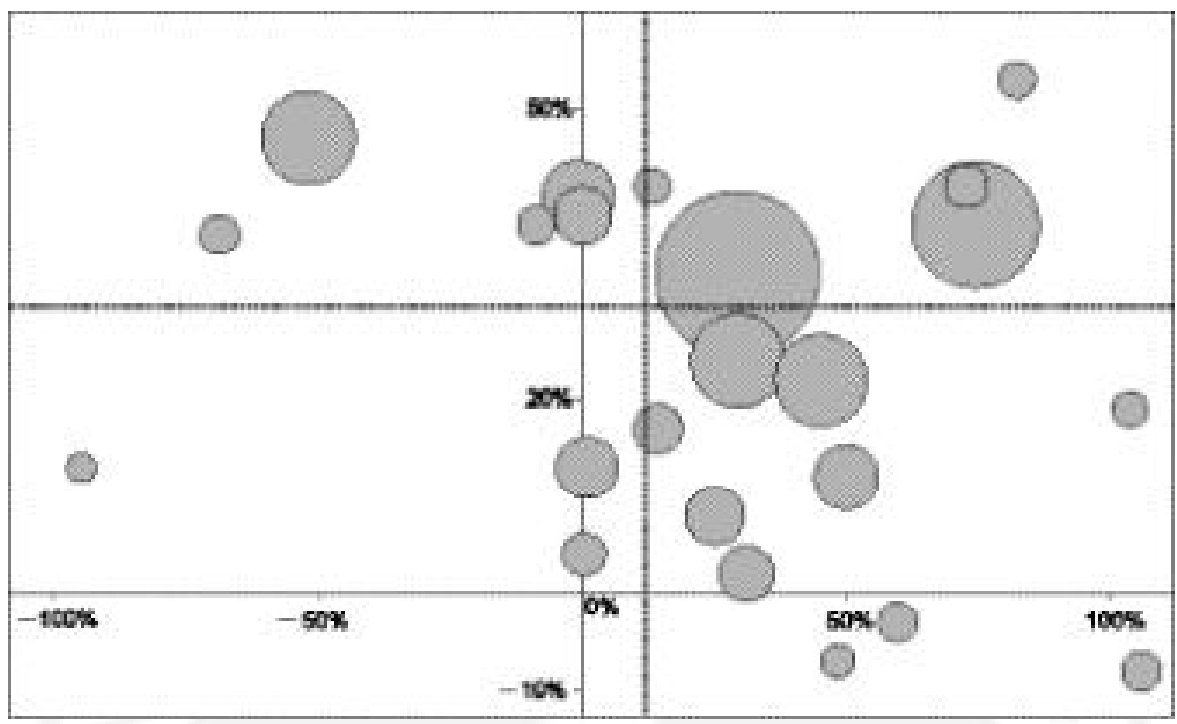

Gráfico 2

Matriz de clasificación para los 25 principales mercados chinos

nas de aproximadamente 140 millones de dólares. Los principales sectores presentes en este cuadrante, en número de subpartidas, son: minería (4), pesca (3) y agropecuario y agroindustrial (3). El tamaño de los mercados demandantes correspondientes -total de importaciones de China- fluctúa entre los 4 mil millones de dólares de la subpartida 260111 y los 543 mil dólares de la subpartida 140410. Además, cada una de las 5 primeras subpartidas de este cuadrante representa un mercado por encima de los 200 millones de dólares ${ }^{12}$.

Por otro lado, si se toma en cuenta el resultado de los indicadores comerciales, 10 de las 12 subpartidas pertenecientes al primer cuadrante presentan un índice de VCR mayor de 1, mientras 5 obtienen un

12. El tamaño del mercado demandante constituye el valor potencial de las exportaciones que un país podría dirigir a China. resultado mayor de 1 en el índice de DCR de las importaciones chinas. Al comparar los resultados, hay una coincidencia en 4 subpartidas. Finalmente, 9 subpartidas presentan un índice de IE superior a 1, lo que indica que las exportaciones del Perú ya poseen cierto nivel de especialización en el mercado chino. Así, 3 subpartidas del primer cuadrante presentan un resultado favorable en los tres índices comerciales en análisis, todas ellas relacionadas con el sector minero. Es necesario resaltar que en el cuadrante en estudio hay algunas subpartidas en las que China presenta una alta participación en el mercado mundial. Este es el caso de las subpartidas $260111^{13}, 440729$ y 740321 , que representan el $57 \%, 48 \%$ y $42 \%$ de las importaciones mundiales, respectivamente.

13. Debe resaltarse que en el caso de esta subpartida, los 3 indicadores comerciales en estudio resultaron favorables. 
Cuadro 8. Matriz de clasificación: primer cuadrante

\begin{tabular}{|c|c|c|c|c|c|c|}
\hline Rank. & $\begin{array}{c}\mathrm{N}^{\circ} \\
\text { Subpartida }\end{array}$ & Subpartida & VCR & DCR & IE & CM \% \\
\hline 1 & 260111 & Minerales de hierro y sus concentrados sin aglomerar. & $\mathrm{x}$ & $\mathrm{x}$ & $\mathrm{x}$ & 57 \\
\hline 2 & 740311 & Cátodos y secciones de cátodos, de cobre refinado. & $x$ & $x$ & $x$ & 25 \\
\hline \multirow[t]{2}{*}{13} & 440729 & Las demás maderas aserradas o desbastadas & & & & \\
\hline & & longitudinalmente. & $\mathrm{x}$ & $\mathrm{x}$ & & 48 \\
\hline 19 & 260700 & Minerales de plomo y sus concentrados. & $x$ & $x$ & $\mathrm{x}$ & 31 \\
\hline 20 & 210690 & Las demás preparaciones alimenticias. & & & & 2 \\
\hline 28 & 790111 & $\begin{array}{l}\text { Cinc en bruto sin alear, con un contenido de cinc, } \\
\text { en peso, superior o igual al } 99,99 \% \text {. }\end{array}$ & $x$ & & $x$ & 4 \\
\hline 29 & 740321 & Aleaciones de cobre en bruto, a base de cobre-cinc (latón) & & $x$ & & 42 \\
\hline 37 & 30759 & Pulpos, congelados, secos, salados o en salmuera. & $\mathrm{x}$ & & $\mathrm{x}$ & 4 \\
\hline \multirow[t]{2}{*}{46} & 30799 & Los demás moluscos e invertebrados secos, & & & & \\
\hline & & salados o en salmuera. & $\mathrm{x}$ & & $\mathrm{x}$ & 2 \\
\hline 54 & 160590 & Los demás crustáceos preparados o conservados. & $\mathrm{x}$ & & $x$ & \\
\hline 61 & 200290 & Los demás tomates preparados o conservados. & $\mathrm{x}$ & & $\mathrm{x}$ & \\
\hline 62 & 140410 & $\begin{array}{l}\text { Materias primas vegetales de las utilizadas } \\
\text { para teñir o curtir. }\end{array}$ & $x$ & & $x$ & 2 \\
\hline
\end{tabular}

\section{b) Segundo cuadrante: oportunidades no aprovechadas}

El segundo cuadrante clasifica a aquellas subpartidas que el Perú exportó a China a una tasa de crecimiento por debajo del promedio de sus exportaciones, y que China demandó del mundo a una tasa de crecimiento mayor al promedio de sus importaciones. Frente a una demanda significativamente creciente de China, lo anterior significa que el Perú no está aprovechando los mercados de dichas subpartidas al exportar cada vez menos o a tasas por debajo del promedio. El número de subpartidas incluidas en este cuadrante es de 8, lo que en el 2003 equivalió a un valor de exportaciones peruanas de alrededor de 60 millones de dólares; 3 de estas subpartidas pertenecen al sector minero. El tamaño de los mercados correspondientes varía entre los 132 millones de dólares de la subpartida 720449 y los 2,6 mi- llones de dólares de la subpartida 280450. Además, cada una de las 6 primeras subpartidas de este cuadrante representa un mercado por encima de los 150 millones de dólares. En este cuadrante, el análisis de los indicadores comerciales muestra que sólo 4 de las 8 subpartidas encontradas presentan un índice de VCR mayor de 1. Por otro lado, mediante el índice de DCR se obtiene que del total de subpartidas del cuadrante, sólo 2 no presentan un resultado mayor de 1. Asimismo, el Perú se encuentra especializado en el mercado chino en 3 de las subpartidas del cuadrante, todas ellas pertenecientes al sector minero (subpartidas 260112, 260800 y 280450). Debe resaltarse, además, que en los tres casos se encuentra un resultado favorable tanto para el índice de VCR como para el de DCR. Asimismo, China representa para esas subpartidas, una participación en el comercio mundial de $21 \%$, $8 \%$ y $15 \%$, respectivamente. 
Cuadro 9. Matriz de clasificación: segundo cuadrante

\begin{tabular}{|c|c|c|c|c|c|c|}
\hline Rank. & $\begin{array}{c}N .^{\circ} \\
\text { Subpartida }\end{array}$ & Subpartida & VCR & DCR & IE & $\begin{array}{l}\text { CM } \\
\%\end{array}$ \\
\hline 4 & 720449 & $\begin{array}{l}\text { Los demás desperdicios y desechos } \\
\text { de hierro o de acero. }\end{array}$ & & $x$ & & 18 \\
\hline 6 & 260112 & $\begin{array}{l}\text { Minerales de hierro y sus concentrados } \\
\text { aglomerados. }\end{array}$ & $x$ & $x$ & $x$ & 21 \\
\hline 10 & 848340 & $\begin{array}{l}\text { Engranajes y ruedas de fricción, excepto las simples } \\
\text { ruedas dentadas y demás órganos elementales. }\end{array}$ & & $x$ & & 8 \\
\hline 16 & 30379 & $\begin{array}{l}\text { Los demás pescados congelados, excluidos filetes, } \\
\text { hígados, huevas y lechas. }\end{array}$ & $x$ & $x$ & & 10 \\
\hline 18 & 440710 & $\begin{array}{l}\text { Madera aserrada o desbastada longitudinalmente } \\
\text { de coníferas. }\end{array}$ & & & & 1 \\
\hline 23 & 260800 & Minerales de cinc y sus concentrados. & $x$ & $x$ & $x$ & 8 \\
\hline 48 & 51191 & $\begin{array}{l}\text { Los demás productos de pescados o de crustáceos, } \\
\text { moluscos u otros invertebrados acuáticos. }\end{array}$ & & & & 5 \\
\hline 57 & 280450 & Boro; teluro. & $x$ & $x$ & $x$ & 15 \\
\hline
\end{tabular}

c) Tercer cuadrante: productos en declive

El tercer cuadrante clasifica a aquellas subpartidas que el Perú exportó a China y que este país importó del mundo, y que en ambos casos crecieron por debajo de sus respectivos promedios. Frente a una demanda decreciente de China, lo anterior significa que las subpartidas exportadas por el Perú representan productos con una oferta y una demanda en declive, dentro de dicho país. El número de subpartidas incluidas en este cuadrante es de 17, lo que en el 2003 equivalió a un valor aproximado de exportaciones peruanas de 3 millones de dólares; 4 de estas subpartidas están relacionadas con las actividades de pesca, 4 corresponden al sector químico y las otras 4 pertenecen al sector textil. El tamaño de los mercados correspondientes varía entre los 558 millones de dólares de la subpartida
440799 y los 2 millones de dólares de la subpartida 151590. Además, cada una de las 10 primeras subpartidas de este cuadrante representa un mercado por encima de los 25 millones de dólares. En lo que respecta al resultado de los indicadores comerciales de las subpartidas pertenecientes a este cuadrante, 10 de ellas presentan un resultado favorable en el índice de VCR. Asimismo, 8 de estas poseen un índice de DCR mayor de 1 para China, coincidiendo en 5 subpartidas los resultados de ambos índices. Por otro lado, en lo que respecta al nivel de especialización de las exportaciones peruanas en el mercado chino, 5 subpartidas presentan un resultado mayor de 1 . De esta forma, 2 subpartidas, la 261390 y la 320290, obtienen un resultado favorable en los tres indicadores utilizados. Finalmente, debe resaltarse que China representa $12 \%$ y $13 \%$ de las importaciones mundiales de estas subpartidas, respectivamente. 
Cuadro 10. Matriz de clasificación: tercer cuadrante

\begin{tabular}{|c|c|c|c|c|c|c|}
\hline Rank. & $\begin{array}{c}N .^{\circ} \\
\text { Subpartida }\end{array}$ & Subpartida & VCR & DCR & IE & CM \% \\
\hline 8 & 440799 & $\begin{array}{l}\text { Las demás maderas tropicales aserradas o } \\
\text { desbastadas longitudinalmente. }\end{array}$ & & $\mathrm{x}$ & & 22 \\
\hline 14 & 391590 & $\begin{array}{l}\text { Desechos, recortes y desperdicios } \\
\text { de los demás plásticos. }\end{array}$ & & $x$ & & 32 \\
\hline 25 & 30613 & Camarones, langostinos, quisquillas. & $\mathrm{x}$ & & & 2 \\
\hline 30 & 730630 & $\begin{array}{l}\text { Tubos de hierro o acero sin alear, soldados, } \\
\text { de sección circular de hierro o acero. }\end{array}$ & & & & 2 \\
\hline 32 & 261390 & Los demás minerales de molibdeno y sus concentrados. & $x$ & $x$ & $x$ & 13 \\
\hline 33 & 847490 & $\begin{array}{l}\text { Partes de máquinas y aparatos para clasificar, cribar, } \\
\text { separar, lavar, quebrantar. }\end{array}$ & & & $x$ & 2 \\
\hline 35 & 320290 & Los demás productos y preparaciones. & $\mathrm{x}$ & $x$ & $x$ & 12 \\
\hline 38 & 30559 & Los demás pescados secos, incluso salado, sin ahumar. & $x$ & $x$ & & 8 \\
\hline 39 & 281000 & Óxido de boro; ácidos bóricos. & $\mathrm{x}$ & $\mathrm{x}$ & & 21 \\
\hline 40 & 511119 & Los demás tejidos de lana o de pelo fino cardado. & & $\mathrm{x}$ & & 8 \\
\hline 44 & 30490 & Las demás carnes de pescado congeladas. & $\mathrm{x}$ & & & 2 \\
\hline 45 & 121190 & Las demás plantas, partes de plantas. & $\mathrm{x}$ & & & 2 \\
\hline 49 & 511190 & Los demás tejidos de lana cardada o de pelo fino cardado. & $\mathrm{x}$ & $\mathrm{x}$ & & 15 \\
\hline 50 & 30420 & Filetes congelados. & $\mathrm{x}$ & & & \\
\hline 55 & 510810 & $\begin{array}{l}\text { Hilados de pelo fino cardado sin acondicionar } \\
\text { para la venta al por menor. }\end{array}$ & & & & \\
\hline 56 & 848030 & Modelos para moldes. & & & $x$ & 2 \\
\hline 58 & 151590 & Los demás aceites y grasas vegetales. & $x$ & & $x$ & \\
\hline
\end{tabular}

\section{d) Cuarto cuadrante: demanda decreciente}

El cuarto cuadrante clasifica a aquellas subpartidas que el Perú exportó a China a una tasa de crecimiento por encima del promedio, y que China demandó del mundo a una tasa de crecimiento menor al del promedio de sus importaciones. Frente a una demanda decreciente de China, lo anterior significa que el Perú está creciendo cada vez más dentro de un mercado que se va haciendo más pequeño. El número de subpartidas incluidas en este cuadrante es de 28, lo que en el 2003 equivalió a un valor de exportaciones peruanas de unos 470 millones de dólares. Entre los sectores con mayor participación en este monto figuran el textil, con 6 subpartidas; el siderúrgico y metalúrgico, con 4 subpartidas, y los sectores agropecuario, minero y químico, cada uno con tres subpartidas. El tamaño de los mercados demandantes correspondientes fluctúa entre los 1333 millones de dólares de la subpartida 740400 y los 56 mil dólares de la subpartida 100590. Además, cada una de las 11 primeras subpartidas de este cuadrante comprende un mercado superior a $150 \mathrm{mi}$ llones de dólares, mientras que cada una de las 6 subpartidas subsiguientes representa un mercado por encima de los 25 millones de dólares. En lo que respecta al resultado de los indicadores comerciales, 
Cuadro 11. Matriz de clasificación: cuarto cuadrante

\begin{tabular}{|c|c|c|c|c|c|c|}
\hline Rank. & $\begin{array}{c}N .^{\circ} \\
\text { Subpartida }\end{array}$ & Subpartida & CR & DCR & IE C & CM \% \\
\hline 3 & 740400 & Desperdicios y desechos de cobre. & & $\mathrm{x}$ & & 38 \\
\hline 5 & 260300 & Minerales de cobre y sus concentrados. & $\mathrm{x}$ & $x$ & $\mathrm{x}$ & 24 \\
\hline 7 & 510111 & Lana esquilada, sucia o lavada en vivo sin cardar ni peinar. & $x$ & $x$ & & 40 \\
\hline 9 & 230120 & $\begin{array}{l}\text { Harina, polvo y pellets de pescado o de crustáceos, } \\
\text { moluscos o de otros invertebrados. }\end{array}$ & $\mathrm{x}$ & $\mathrm{x}$ & $\mathrm{x}$ & 26 \\
\hline 11 & 760200 & Aluminio, desechos y desperdicios. & & $x$ & $x$ & 12 \\
\hline 12 & 842230 & $\begin{array}{l}\text { Máquinas y aparatos para llenar, cerrar, capsular } \\
\text { o etiquetar botellas, cajas, sacos. }\end{array}$ & & & $x$ & 9 \\
\hline 15 & 740200 & $\begin{array}{l}\text { Cobre sin refinar; ánodos de cobre para } \\
\text { refinado electrolítico. }\end{array}$ & $\mathrm{x}$ & $\mathrm{x}$ & $\mathrm{x}$ & 16 \\
\hline 17 & 390760 & Politereftalato de etileno. & & & $x$ & 6 \\
\hline 21 & 790120 & Aleaciones de cinc en bruto. & $x$ & $x$ & $x$ & 35 \\
\hline 22 & 510529 & Las demás lanas peinadas, excepto a granel. & $x$ & $x$ & $x$ & 12 \\
\hline 24 & 510710 & $\begin{array}{l}\text { Hilados de lana peinada sin acondicionar para } \\
\text { la venta al por menor. }\end{array}$ & $\mathrm{x}$ & $\mathrm{x}$ & & 13 \\
\hline 26 & 520532 & $\begin{array}{l}\text { Hilados retorcidos o cableados, de fibras sin peinar } \\
\text { de algodón de título inferior a } 714,2 \text {. }\end{array}$ & & $x$ & & 36 \\
\hline 27 & 30749 & $\begin{array}{l}\text { Jibias, globitos, calamares y potas, congelados, } \\
\text { secos, salados o en salmuera. }\end{array}$ & $\mathrm{x}$ & $\mathrm{x}$ & $\mathrm{x}$ & 8 \\
\hline 31 & 842290 & $\begin{array}{l}\text { Partes de máquinas y aparatos para limpiar, secar, } \\
\text { llenar o cerrar botellas. }\end{array}$ & & & & 2 \\
\hline 34 & 720421 & Desperdicios y desechos de fundición de acero inoxidable. & & & & 1 \\
\hline 36 & 740829 & Los demás alambres de cobre. & $x$ & $\mathrm{x}$ & & 20 \\
\hline 41 & 550630 & $\begin{array}{l}\text { Fibras sintéticas discontinuas, cardadas, peinadas } \\
\text { o transformadas de otro modo. }\end{array}$ & $x$ & $x$ & & 35 \\
\hline 42 & 511290 & Los demás tejidos de lana peinada o de pelo fino peinado. & $x$ & $x$ & & 20 \\
\hline 43 & 121220 & Algas, frescas o secas, incluso pulverizadas. & $x$ & $x$ & $\mathrm{x}$ & 7 \\
\hline 47 & 320300 & $\begin{array}{l}\text { Materias colorantes de origen vegetal o animal, } \\
\text { aunque sean de constitución química definida. }\end{array}$ & $\mathrm{x}$ & & $\mathrm{x}$ & 3 \\
\hline 51 & 150420 & $\begin{array}{l}\text { Aceites y grasas de pescado y sus fracciones, } \\
\text { excepto los aceites de hígado. }\end{array}$ & $\mathrm{x}$ & & $\mathrm{x}$ & 2 \\
\hline 52 & 440920 & Maderas perfiladas longitudinalmente de no coníferas. & $x$ & & $\mathrm{x}$ & \\
\hline 53 & 510820 & $\begin{array}{l}\text { Hilados de pelo fino peinado sin acondicionar } \\
\text { para la venta al por menor. }\end{array}$ & $\mathrm{x}$ & $\mathrm{x}$ & $\mathrm{x}$ & 7 \\
\hline 59 & 440724 & $\begin{array}{l}\text { Maderas aserrada o desbastadas longitudinalmente } \\
\text { de virola, mahogany. }\end{array}$ & $\mathrm{x}$ & & $\mathrm{x}$ & 1 \\
\hline 60 & 282741 & Oxicloruro e hidroxicloruros de cobre. & $x$ & & $x$ & 3 \\
\hline 63 & 510320 & Los demás desperdicios de lana o de pelo fino. & $\mathrm{x}$ & & $\mathrm{x}$ & \\
\hline 64 & 110620 & $\begin{array}{l}\text { Harina y sémola de sagu o de las raíces o } \\
\text { tubérculos de la partida } 07.14 \text {. }\end{array}$ & $\mathrm{x}$ & & $\mathrm{x}$ & \\
\hline 65 & 100590 & Los demás maíces. & & & $x$ & \\
\hline
\end{tabular}


se observa que 20 subpartidas presentan un valor mayor de 1 en el índice de VCR, 18 subpartidas obtienen un resultado favorable en el índice de DCR para China; ambos resultados coinciden en 13 subpartidas. Por otro lado, 10 subpartidas presentan un índice de especialización mayor de 1, mientras 8 obtienen un resultado mayor de 1 en los tres indicadores en estudio.

\subsubsection{Primera matriz de}

\section{potencialidades: los principales mercados de exportación nacional}

Para la elaboración de esta matriz se consideró sólo aquellas subpartidas nacionales que no hubiesen sido exportadas a China en el periodo 1999-2003 y de las cuales se hubiesen exportado al mundo, en promedio, más de 50 mil dólares por año. Además, se tomó en cuenta sólo aquellas que durante ese mismo periodo se hubiesen exportado por lo menos dos años. De esa forma, el número total de subpartidas resultantes es de 1049.

Para proceder a graficar los resultados, se debe calcular lo siguiente: a) para el eje «X», la tasa de crecimiento de las subpartidas exportadas por el Perú al mundo y que no se exporten a China, b) para el eje «y», la tasa de crecimiento de las subpartidas importadas por China desde el mundo pero no desde el Perú, c) para señalar el tamaño del mercado, las importaciones totales chinas del 2003 y d) para establecer la separación de los cuadrantes, las tasas de crecimiento promedio de las exportaciones peruanas y de las importaciones chinas hacia y desde el mundo, respectivamente. Una vez calculados

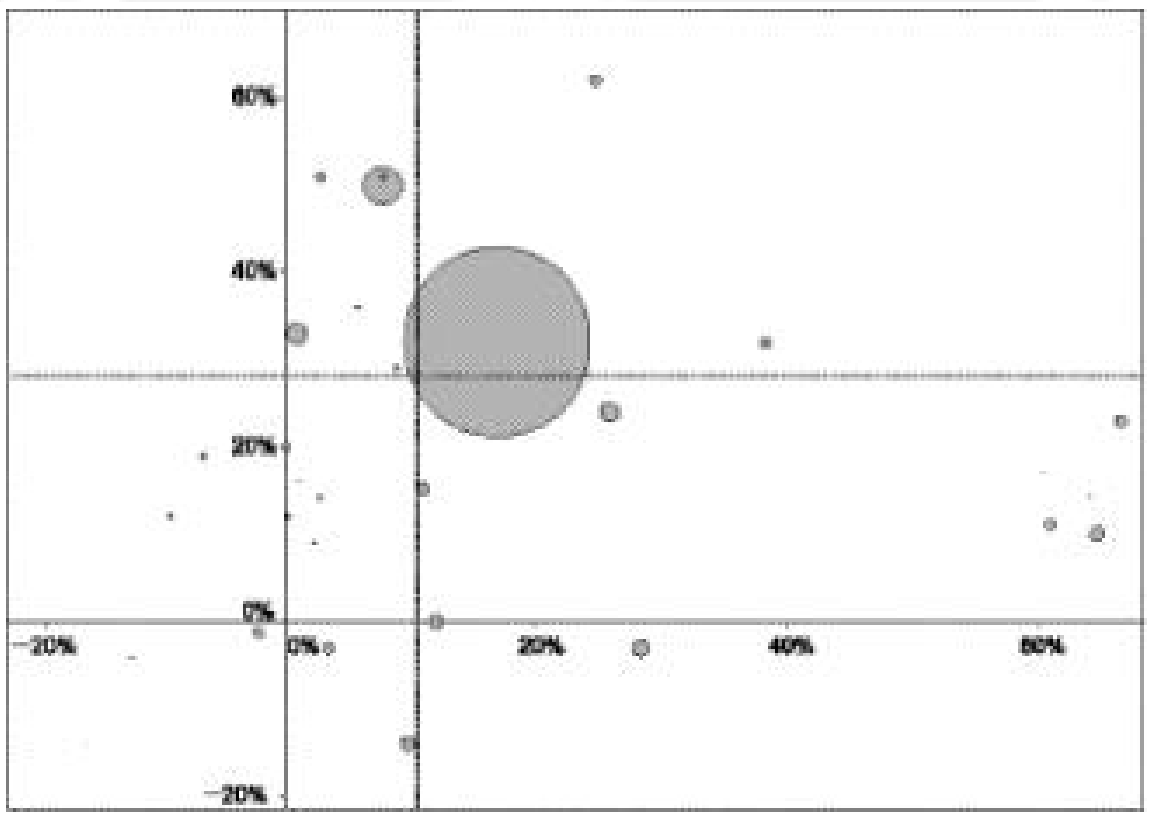

Gráfico 3

Primera matriz de potencialidades para los 40 principales mercados de las exportaciones peruanas 
estos valores, se estableció un ranking de los 40 mercados más grandes para las exportaciones peruanas entre 1999 y el 2003. La matriz de potencialidades para estas subpartidas se presenta en el gráfico $3^{14}$.

\section{a) Primer cuadrante: mercados con} obstáculos para productos de exportación estrella

Este primer cuadrante clasifica a aquellas subpartidas que el Perú exportó al mundo pero no a China, y las que este país importó del mundo pero no del Perú, y que en ambos casos crecieron por encima de sus respectivos promedios. Lo anterior significa que el Perú podría aprovechar los mercados de dichas subpartidas, ya que exportaría cada vez más frente a una demanda creciente en China. El número de subpartidas incluidas en este cuadrante es de 6, lo que en el 2003 equivalió a un valor de exportaciones peruanas de unos 384 millones de dólares. De este grupo, 2 subpartidas pertenecen al sector minero y otras 2 corresponden al sector químico. El tamaño de los mercado potenciales -total de importaciones de China- fluc- túa entre los 19782 millones de dólares de la subpartida 270900 y los 946 mil dólares de la subpartida 261610. En total, sólo 3 subpartidas de este cuadrante representan, cada una, un mercado por encima de los 30 millones de dólares (las restantes no superan los 3 millones de dólares). En lo referido al resultado de los indicadores comerciales, debe resaltarse que el total de las subpartidas pertenecientes a este cuadrante presentan un índice de VCR mayor de 1, resultado que coincide en un caso con el encontrado para el índice de DCR para China: subpartida 270900. Debe resaltarse, además, que las importaciones chinas de esta subpartida representan el $10 \%$ del comercio mundial. Para el resto de subpartidas, la participación de las importaciones chinas en el comercio mundial es menor de la unidad porcentual.

b) Segundo cuadrante: mercados con obstáculos para productos de demanda creciente

El segundo cuadrante clasifica a aquellas subpartidas que el Perú exportó al mun-

Cuadro 12. Primera matriz de potencialidades: primer cuadrante

\begin{tabular}{rrllc}
\hline Rank. & $\begin{array}{c}\text { N. }^{\circ} \\
\text { Subpartida }\end{array}$ & \multicolumn{1}{c}{ Subpartida } & VCR & DCR CM \% \\
\hline 3 & 270900 & Aceites crudos de petróleo o de minerales bituminosos. & X & X \\
11 & 392330 & Bombonas, botellas, frascos y artículos similares. & X & \\
13 & 261610 & Minerales de plata y sus concentrados. & X \\
22 & 401120 & Neumáticos nuevos de caucho del tipo utilizado en & & \\
& & autobuses y camiones. & & \\
24 & 200590 & Las demás legumbres u hortalizas y las mezclas de & X \\
& & hortalizas y/o legumbres preparadas. & X \\
\hline
\end{tabular}

14. Las siguientes subpartidas no han sido incorporadas en el gráfico 3 con la finalidad de que la escala permita una mejor percepción del mismo: 40291, 261690, 261610, 70310, 260900, 611120, 71080, 200590, 800110, 160413. 
Cuadro 13. Primera matriz de potencialidades: segundo cuadrante

\begin{tabular}{|c|c|c|c|c|c|}
\hline Rank. & $\begin{array}{c}N^{\circ} .^{\circ} \\
\text { Subparti }\end{array}$ & Subpartida & VCR & DCR & $\begin{array}{c}\text { CM } \\
\%\end{array}$ \\
\hline 2 & 710691 & Plata en bruto. & $x$ & & 1 \\
\hline 4 & 610510 & Camisas de punto de algodón, para hombres o niños & $x$ & & \\
\hline 5 & 800110 & Estaño en bruto, sin alear. & $x$ & & 3 \\
\hline 6 & 611020 & $\begin{array}{l}\text { Suéteres, jerséis, pullovers, cardigans, chalecos y } \\
\text { artículos similares de algodón. }\end{array}$ & $\mathrm{x}$ & & 2 \\
\hline 8 & 740811 & $\begin{array}{l}\text { Alambres de cobre refinado en el que la mayor } \\
\text { dimensión de la sección transversal sea superior. }\end{array}$ & $x$ & $x$ & 16 \\
\hline 12 & 80450 & Guayabas, mangos y mangostanes, frescos o secos. & $\mathrm{x}$ & & 4 \\
\hline 29 & 70310 & Cebollas y chalotes, frescos o refrigerados. & $x$ & & \\
\hline 32 & 610462 & $\begin{array}{l}\text { Pantalones, pantalones con peto y pantalones cortos } \\
\text { de punto de algodón, para mujeres. }\end{array}$ & $x$ & & \\
\hline
\end{tabular}

do, aunque no a China, a una tasa de crecimiento por debajo del promedio, pero que China demandó del mundo, menos del Perú, a una tasa de crecimiento mayor al promedio de sus importaciones. Lo anterior significa que el Perú, al no tener acceso a los mercados de dichas subpartidas, no los está aprovechando y, probablemente por ello, tiene una fuerza exportadora cada vez menor. El número de subpartidas incluidas en este cuadrante es de 8, lo que en el 2003 equivalió a un valor de exportaciones peruanas de alrededor de 496 millones de dólares. De estas subpartidas, 3 corresponden a prendas de vestir, 2 pertenecen a los sectores agropecuario y agroindustrial, otras 2 están referidas al sector minero y 1 corresponde al sector siderúrgico y metalúrgico. El tamaño de los mercados potenciales de demanda fluctúa entre los 564 millones de dólares de la subpartida 740811 y los 336 mil dólares de la subpartida 070310. En total, sólo 4 subpartidas de este cuadrante representan, cada una, un mercado por encima de los 20 millones de dólares. En lo que respecta al resultado de los indicadores comerciales, se observa que, al igual que en el cuadrante anterior, todas las subpartidas de la muestra presentan un índice de VCR mayor de 1. Sin embargo, este resultado resulta opuesto al del indicador de DCR para China, pues sólo una subpartida, la 740811, obtiene un resultado mayor de 1 . Asimismo, debe resaltarse, a partir del análisis de la cuota de mercado chino, que este país no presenta una alta participación en el comercio mundial de ninguna de las subpartidas del cuadrante. La mayor, ya mencionada, representa el $16 \%$ del mercado mundial.

\section{c) Tercer cuadrante: productos en declive}

El tercer cuadrante clasifica a aquellas subpartidas que el Perú exportó al mundo, aunque no a China, y que el país oriental importó del mundo, menos del Perú. Además, se trata de las subpartidas que crecieron por debajo de sus respectivos promedios o que están decreciendo. Lo anterior significa que las subpartidas exportadas por el Perú (a tasas de crecimiento por debajo del promedio o negativas), lo hacen a mercados con una demanda en 
Cuadro 14. Primera matriz de potencialidades: tercer cuadrante

\begin{tabular}{|c|c|c|c|c|c|}
\hline Rank. & $\begin{array}{c}N^{\circ} \\
\text { Subpartidas }\end{array}$ & Subpartida & VCR & DCR & $\begin{array}{l}\text { CM } \\
\%\end{array}$ \\
\hline 1 & 90111 & Café sin tostar, sin descafeinar. & $x$ & & \\
\hline 10 & 790500 & Chapas, hojas y bandas, de cinc. & $x$ & & 4 \\
\hline 14 & 260900 & Minerales de estaño y sus concentrados. & & $\mathrm{x}$ & 9 \\
\hline 15 & 30729 & $\begin{array}{l}\text { Veneras, volandeiras otros, congelados, secos, } \\
\text { salados o en salmuera. }\end{array}$ & $\mathrm{x}$ & & \\
\hline 16 & 71080 & Las demás legumbres y hortalizas. & $x$ & & \\
\hline 18 & 160420 & Las demás preparaciones y conservas de pescados. & $x$ & & \\
\hline 25 & 620342 & $\begin{array}{l}\text { Pantalones, pantalones con peto y pantalones } \\
\text { cortos de algodón. }\end{array}$ & & & \\
\hline 26 & 320500 & $\begin{array}{l}\text { Lacas colorantes; preparaciones a que se refiere } \\
\text { la nota } 3 \text { de este capítulo, a base de laca. }\end{array}$ & $\mathrm{x}$ & & 5 \\
\hline 27 & 180400 & Manteca, grasa y aceite de cacao. & $\mathrm{x}$ & & \\
\hline 28 & 740710 & Barras y perfiles de cobre refinado. & $x$ & $x$ & 12 \\
\hline 33 & 160413 & $\begin{array}{l}\text { Sardinas, sardinelas y espadines en conserva, } \\
\text { entero o en trozos. }\end{array}$ & $\mathrm{x}$ & & \\
\hline 34 & 340220 & $\begin{array}{l}\text { Preparaciones tenso activas acondicionadas para } \\
\text { la venta al por menor. }\end{array}$ & $\mathrm{x}$ & & \\
\hline 36 & 740821 & Alambre de aleaciones a base de cobre. & $x$ & $x$ & 7 \\
\hline 37 & 240220 & Cigarrillos que contengan tabaco. & & & \\
\hline 38 & 740312 & Barras para alambrón (wire-bars), de cobre refinado. & $x$ & & 1 \\
\hline
\end{tabular}

declive dentro de dicho país, por lo que para el Perú son potencialmente menos relevantes como mercados de destino. El número de subpartidas incluidas en este cuadrante es de 15 , lo que en el 2003 equivalió a un valor de exportaciones peruanas de alrededor de 342 millones de dólares. De estas 15, la mayoría de subpartidas pertenece a los sectores agropecuario y agroindustrial, pesca y sectores metalúrgico y siderúrgico, con 3 subpartidas cada uno. El tamaño de los mercados correspondientes varía entre los 86 millones de dólares de la subpartida 740710 y los 49 mil dólares de la subpartida 160413. En total, 13 subpartidas de este cuadrante representan, cada una, un mercado por debajo de los 20 millones de dólares. Por otro lado, se observa que casi la totalidad de las subpartidas de este cuadrante presentan un resultado mayor de 1 en el índice de VCR; las excepciones son las subpartidas 260900, 620342 y 240220. Esto da indicios de un factor de eficiencia que el Perú podría aprovechar a través de la concesión de una preferencia arancelaria por parte de China. Sin embargo, el índice de DCR para China revela que este país es considerado un gran importador sólo en tres de las subpartidas, de las cuales sólo dos coinciden en resultados favorables para ambos índices: las subpartidas 740710 y 740821 , que pertenecen al sector siderúrgico y metalúrgico. Es necesario destacar que, en el caso de la subpartida 740710, China posee una participación en el comercio mundial de $12 \%$, la mayor presentada entre las subpartidas del cuadrante. 
d) Cuarto cuadrante: mercados con obstáculos a los productos con demanda decreciente

El cuarto cuadrante clasifica a aquellas subpartidas que el Perú exportó al mundo, aunque no a China, a una tasa de crecimiento por encima del promedio de sus exportaciones, y que China demandó del mundo, menos del Perú, a una tasa de crecimiento menor al promedio de sus importaciones. Lo anterior significa que el Perú podría crecer dentro de un mercado cada vez más pequeño. El número de subpartidas incluidas en este cuadrante es de 11, lo que en el 2003 equivalió a un valor de exportaciones peruanas de alrededor de 274 millones de dólares. De estas subpartidas, el sector con mayor participación es el agropecuario y agroindustrial, con 4 subpartidas, y el de las prendas de vestir, con 2 subpartidas. El tamaño de los mercados demandantes correspondientes fluctúa entre los $135 \mathrm{mi}$ llones de dólares de la subpartida 170111 y los 766 mil dólares de la subpartida 611120. En total, siete subpartidas de este cuadrante representan, cada una, un mercado por encima de los 40 millones de dólares. Finalmente, en lo que respecta al análisis de los indicadores comerciales, se observa que el Perú posee un índice de VCR mayor de 1 en todas las subpartidas pertenecientes al cuadrante; sin embargo, China presenta una baja participación en las importaciones mundiales de estas subpartidas, lo cual se revela mediante el resultado menor de 1 en el índice de DCR para todas las subpartidas del cuadrante y a través del análisis de sus cuotas de mercado: en todos los casos menores de $4 \%$.

Cuadro 15. Primera matriz de potencialidades: cuarto cuadrante

\begin{tabular}{|c|c|c|c|c|c|}
\hline Rank. & $\begin{array}{c}\mathrm{N}^{0} \\
\text { Subpartida }\end{array}$ & Subpartida & VCR & DCR & $\begin{array}{c}\mathrm{CM} \\
\% \\
\end{array}$ \\
\hline 7 & 711319 & $\begin{array}{l}\text { Joyería de otros metales preciosos, incluso } \\
\text { revestidos o chapados de metales preciosos. }\end{array}$ & $x$ & & \\
\hline 9 & 610610 & $\begin{array}{l}\text { Camisas, blusas, blusas camiseras y polos, de punto } \\
\text { de algodón, para mujeres o niñas. }\end{array}$ & $\mathrm{x}$ & & \\
\hline 17 & 170111 & $\begin{array}{l}\text { Azúcar de caña en bruto sin aromatizar ni colorear, } \\
\text { en estado sólido. }\end{array}$ & $x$ & & 4 \\
\hline 19 & 90420 & Pimientos secos, triturados o pulverizados (pimentón). & $\mathrm{x}$ & & \\
\hline 20 & 80610 & Uvas frescas. & $\mathrm{x}$ & & 1 \\
\hline 21 & 490199 & Los demás libros, folletos e impresos similares. & $x$ & & \\
\hline 23 & 611120 & $\begin{array}{l}\text { Prendas y complementos de vestir, de punto } \\
\text { de algodón, para bebes. }\end{array}$ & $x$ & & \\
\hline 31 & 230990 & $\begin{array}{l}\text { Las demás preparaciones del tipo utilizadas para } \\
\text { la alimentación animal. }\end{array}$ & $\mathrm{x}$ & & 2 \\
\hline 35 & 330499 & Las demás preparaciones de maquillaje. & $\mathrm{x}$ & & \\
\hline 39 & 721420 & $\begin{array}{l}\text { Barras hierro con muescas, cordones, huecos } \\
\text { o relieve obtenidos durante el laminado. }\end{array}$ & $\mathrm{x}$ & & 2 \\
\hline 40 & 40291 & Las demás leches sin azucarar ni edulcorar de otro modo. & $x$ & & \\
\hline
\end{tabular}




\subsubsection{Segunda matriz de potencialida- des: los principales mercados según indicadores comerciales}

Además de las consideraciones tomadas para la construcción de la primera matriz de potencialidades, en este caso se consideró sólo a aquellas subpartidas en las que el Perú y China, en el 2003, hubiesen obtenido índices mayores de 1 de VCR y DCR, respectivamente. De esa forma, el número total de subpartidas resultantes es de sólo 52. Luego, se estableció un ranking de los mercados potenciales de destino más grandes para las exportaciones peruanas. Así, la segunda matriz de potencialidades es la que se presenta en el gráfico $4^{15}$.
Como se señaló anteriormente, los cuadrantes quedan definidos por el crecimiento promedio anual de las exportaciones peruanas al mundo y por el crecimiento promedio de las importaciones chinas desde el mundo, ambos para el periodo 1999-2003. Cabe resaltar que sólo 4 subpartidas incluidas en esta matriz se encuentran dentro de la matriz del acápite anterior y que contiene a las 40 subpartidas más exportadas del Perú al resto del mundo para el periodo 1999-2003. Luego, estableciendo un ranking según el tamaño del mercado de destino potencial para las exportaciones peruanas, la clasificación de la totalidad de subpartidas (52) se muestra a continuación.

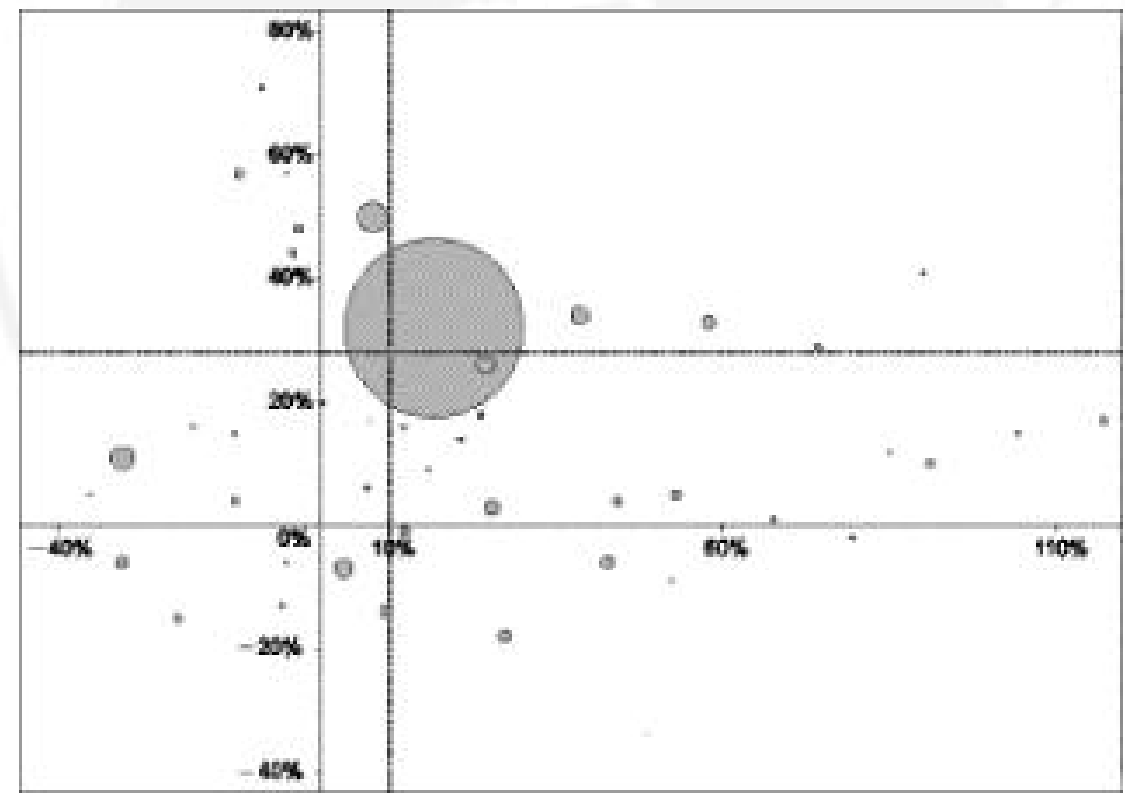

Gráfico 4

Segunda matriz de potencialidad según indicadores comerciales

15. Las siguientes subpartidas no han sido incorporadas en el gráfico 4 con la finalidad de que la escala permita una mejor percepción del mis- mo: 30624, 842520, 284329, 551512, 391530, 520941,870540 . 
a) Primer cuadrante: mercados con obstáculos para productos de exportación estrella

El número de subpartidas incluidas en el primer cuadrante es de 9, lo que en el 2003 equivalió a un valor de exportaciones peruanas de alrededor de 273 millones de dólares. De estas subpartidas, los sectores con mayor participación son el de pesca y el químico, con 2 subpartidas cada uno. El tamaño de los mercados de demanda potencial -total de importaciones de China- varía entre los 19782 millones de dólares de la subpartida 270900 y los 6 millones de dólares de la subpartida 520210. Además, 2 subpartidas de este cuadrante representan, cada una, un mercado por encima de los 130 millones de dólares, mientras que otras 4 representan, cada una, un mercado mayor a los $50 \mathrm{mi}-$ llones de dólares.

\section{b) Segundo cuadrante: mercados con obstáculos para productos de demanda creciente}

El número de subpartidas incluidas en este cuadrante es de 6, lo que en el 2003 equivalió a un valor de exportaciones perua-

Cuadro 16: Segunda matriz de potencialidades: primer cuadrante

\begin{tabular}{|c|c|c|c|c|}
\hline Rank. & $\begin{array}{c}\mathrm{N}^{\circ} \\
\text { Subpartida }\end{array}$ & Subpartida & $\begin{array}{l}\text { M China } \\
2003\end{array}$ & $\begin{array}{c}\text { CM } \\
\%\end{array}$ \\
\hline 1 & 270900 & Aceites crudos de petróleo o de minerales bituminosos. & 19782400 & 10 \\
\hline 5 & 740911 & $\begin{array}{l}\text { Cobre refinado, chapas, planchas, hojas y tiras } \\
\text { enrolladas de espesor superior a } 0,15 \mathrm{~mm} \text {. }\end{array}$ & 138671 & 14 \\
\hline 6 & 520839 & $\begin{array}{l}\text { Los demás tejidos de algodón teñidos de gramaje } \\
\text { inferior o igual a } 200 \mathrm{~g} / \mathrm{m}^{2} \text {. }\end{array}$ & 136054 & 22 \\
\hline 9 & 30624 & Cangrejos de mar, sin congelar. & 81628 & 31 \\
\hline 10 & 30380 & Hígados, huevas y lechas de pescados, congelados. & 71421 & 12 \\
\hline 15 & 391530 & $\begin{array}{l}\text { Desechos, recortes y desperdicios, de polímetros } \\
\text { de cloruro de vinilo. }\end{array}$ & 53638 & 66 \\
\hline 16 & 870540 & Camiones-hormigonera. & 51509 & 14 \\
\hline 28 & 282550 & Óxido e hidróxido de cobre. & 21030 & 20 \\
\hline 42 & 520210 & Desperdicios de hilados. & 5945 & 16 \\
\hline
\end{tabular}

Cuadro 17. Segunda matriz de potencialidades: segundo cuadrante

\begin{tabular}{cclrr}
\hline Rank. & $\begin{array}{c}\mathbf{N}^{\circ} \\
\text { Subpartida }\end{array}$ & \multicolumn{1}{c}{ Subpartida } & $\begin{array}{c}\text { M China } \\
\text { 2003 }\end{array}$ & $\begin{array}{c}\text { CM } \\
\%\end{array}$ \\
\hline 2 & 740811 & $\begin{array}{l}\text { Alambres de cobre refinado en el que la mayor } \\
\text { dimensión de la sección transversal sea superior. }\end{array}$ & 564586 & 16 \\
17 & 280700 & Ácido sulfúrico; óleum. & 43530 & 15 \\
23 & 80290 & $\begin{array}{l}\text { Los demás frutos de cáscara, frescos o secos, } \\
\text { incluso sin cáscara. }\end{array}$ & 28291 & 6 \\
27 & 520622 & Hilados sencillos de fibras peinadas de algodón & 23211 & 43 \\
32 & 252890 & Le título inferior a 714,29 dtex. & 12099 & 17 \\
49 & 382420 & Ĺcidom demás boratos naturales y sus concentrados. & 2731 & 13 \\
\hline
\end{tabular}


nas de alrededor de 50 millones de dólares. En estas subpartidas están presentes, con una subpartida cada uno, los sectores agropecuario y agroindustrial, minero no metálico, petrolero, químico, siderúrgico y metalúrgico, y textil. El tamaño de los mercados potenciales de demanda fluctúa entre los 564 millones de dólares de la subpartida 740811 y los 2,7 millones de dólares de la subpartida 382420. Además, las 4 subpartidas restantes de este cuadrante representan, cada una, un mercado de entre 44 y 12 millones de dólares.

\section{c) Tercer cuadrante: productos en declive}

El número de subpartidas incluidas en este cuadrante es de 14, lo que en el 2003 equivalió a un valor de exportaciones peruanas de alrededor de 29 millones de dólares. De estas subpartidas, 5 pertenecen al sector textil, 4 corresponden al químico, siderúrgico y metalúrgico y 1 está relacionada con el pesquero. El tamaño de los mercados de demanda varía entre los 250 millones de la subpartida 511219 y los 2 millones de dólares la subpartida 401692. Además, 8 subpartidas representan, cada una, un mercado por debajo de los 20 millones de dólares.

\section{d) Cuarto cuadrante: mercados con} obstáculos a los productos con demanda decreciente

El número de subpartidas incluidas en este cuadrante es de 23, lo que en el 2003 equivalió a un valor de exportaciones peruanas que bordea los 30 millones de dólares. Los sectores con mayor participación son el textil (9 subpartidas), el

\section{Cuadro 18. Segunda matriz de potencialidades: tercer cuadrante}

\begin{tabular}{rrlrr}
\hline Rank. & $\begin{array}{c}\text { No } \\
\text { Subpartida }\end{array}$ & \multicolumn{1}{c}{ Subpartida } & $\begin{array}{c}\text { M China } \\
\mathbf{2 0 0 3}\end{array}$ & $\begin{array}{c}\text { CM } \\
\%\end{array}$ \\
\hline 3 & 511219 & Los demás tejidos de lana o de pelo fino peinado. & 249348 & 24 \\
4 & 740819 & Los demás alambres de cobre refinado. & 222000 & 36 \\
8 & 740710 & Barras y perfiles de cobre refinado. & 85556 & 12 \\
13 & 730690 & Los demás tubos y perfiles huecos soldados, remachados, & & \\
& & grapados, de hierro o acero. & 62701 & 14 \\
25 & 510720 & Hilados de lana peinada sin acondicionar para la venta & 27311 & 9 \\
& & al por menor. & & \\
26 & 551012 & Hilados retorcidos o cableados con un contenido de & 25475 & 30 \\
& & fibras artificiales discontinuas. & 17488 & 7 \\
30 & 740821 & Alambre de aleaciones a base de cobre. & 12011 & 6 \\
34 & 500790 & Los demás tejidos de seda o de desperdicios de seda. \\
39 & 320190 & Los demás extractos curtientes de origen vegetal. & 8851 & 15 \\
40 & 30375 & Escualos congelados, excluidos filete, hígado, huevas y lechas. & 7959 & 12 \\
41 & 283325 & Sulfato de cobre. & 7863 & 8 \\
44 & 520541 & Hilados retorcidos o cableados, de fibras peinadas & & \\
& & de título superior o igual a 714,29 dte. & 4240 & 14 \\
47 & 283323 & Sulfato de cromo. & 3055 & 9 \\
48 & 401692 & Gomas de borrar de caucho vulcanizado sin endurecer. & 2885 & 7 \\
\hline
\end{tabular}


siderúrgico-metalúrgico y el metal-mecánico (4 subpartidas cada uno). El tamaño de los mercados potenciales fluctúa entre los 102 millones de dólares de la subpartida 520512 y los 782 mil dólares de la subpartida 280540. En total, 4 subparti- das de este cuadrante representan, cada una, un mercado que supera los 50 millones de dólares, mientras 13 subpartidas están, cada una, por debajo de los 20 millones de dólares.

\section{Cuadro 19. Segunda matriz de potencialidades: cuarto cuadrante}

\begin{tabular}{|c|c|c|c|c|}
\hline Rank. & $\begin{array}{r}N .^{\circ} \\
\text { Subpa }\end{array}$ & Subpartida & $\begin{array}{l}\text { M China } \\
2003 \\
\end{array}$ & $\begin{array}{c}\text { CM } \\
\%\end{array}$ \\
\hline 7 & 520512 & $\begin{array}{l}\text { Hilados sencillos de fibras sin peinar, de título inferior } \\
\text { a } 714,29 \text { dte. }\end{array}$ & 102047 & 9 \\
\hline 11 & 441299 & $\begin{array}{l}\text { Las demás maderas contrachapadas, madera chapada } \\
\text { y madera estratificada. }\end{array}$ & 64935 & 13 \\
\hline 12 & 740919 & $\begin{array}{l}\text { Chapas y bandas de cobre refinado, de espesor superior } \\
\text { a } 0,15 \mathrm{~mm} \text {, sin enrollar. }\end{array}$ & 63885 & 20 \\
\hline 14 & 848490 & $\begin{array}{l}\text { Juegos o surtidos de juntas distinta composición, } \\
\text { presentados en bolsitas, sobres o envases. }\end{array}$ & 59466 & 9 \\
\hline 18 & 841090 & $\begin{array}{l}\text { Partes, incluidos los reguladores de turbinas y ruedas } \\
\text { hidráulicas. }\end{array}$ & 37824 & 9 \\
\hline 19 & 520941 & $\begin{array}{l}\text { Tejidos de algodón con hilados de distintos colores, } \\
\text { de ligamento tafetán de gramaje superior. }\end{array}$ & 36024 & 37 \\
\hline 20 & 740729 & Las demás barras y perfiles de aleaciones de cobre. & 32237 & 15 \\
\hline 21 & 410221 & Pieles en bruto de ovino sin lana (depiladas), piqueladas. & 31262 & 13 \\
\hline 22 & 521041 & $\begin{array}{l}\text { Tejidos de algodón con hilados de distintos colores } \\
\text { de ligamento tafetán, mezclados. }\end{array}$ & 30044 & 12 \\
\hline 24 & 551512 & Tejidos de fibras discontinuas de poliéster. & 28172 & 11 \\
\hline 29 & 550999 & $\begin{array}{l}\text { Los demás hilados de fibras sintéticas discontinuas } \\
\text { sin acondicionar para la venta. }\end{array}$ & 18087 & 41 \\
\hline 31 & 790390 & Los demás polvo y partículas, de cinc. & 16362 & 20 \\
\hline 33 & 71040 & Maíz dulce. & 12014 & 6 \\
\hline 35 & 521049 & $\begin{array}{l}\text { Los demás tejidos de algodón con hilados de } \\
\text { distintos colores. }\end{array}$ & 11130 & 8 \\
\hline 36 & 284329 & Los demás compuestos de plata, excepto el nitrato de plata. & 10762 & 13 \\
\hline 37 & 560819 & $\begin{array}{l}\text { Las demás redes de mallas confeccionadas de } \\
\text { materias textiles. }\end{array}$ & 10748 & 6 \\
\hline 38 & 520841 & $\begin{array}{l}\text { Tejidos de algodón con hilados de distintos colores, } \\
\text { de ligamento tafetán o de gramaje. }\end{array}$ & 10569 & 17 \\
\hline 43 & 741510 & $\begin{array}{l}\text { Puntas y clavos, chinchetas, grapas apuntadas } \\
\text { y similares, de cobre. }\end{array}$ & 5722 & 17 \\
\hline 45 & 681290 & Las demás manufacturas de amianto. & 4150 & 9 \\
\hline 46 & 520853 & $\begin{array}{l}\text { Tejidos de algodón estampados, de ligamento sarga } \\
\text { o cruzado de curso no superior a } 4 \text {. }\end{array}$ & 3535 & 8 \\
\hline 50 & 842520 & $\begin{array}{l}\text { Tornos para ascenso y descenso de jaulas o } \\
\text { montacargas en pozos de minas. }\end{array}$ & 2257 & 14 \\
\hline 51 & 810790 & Las demás manufacturas de cadmio. & 2118 & 24 \\
\hline 52 & 280540 & Mercurio. & 782 & 6 \\
\hline
\end{tabular}




\section{Conclusiones y recomendaciones de política}

A lo largo de la presente investigación se ha resaltado la importancia de la economía china y del comercio bilateral entre China y el Perú. En un contexto en el cual se podría negociar un acuerdo comercial entre ambos países, es muy relevante diseñar herramientas y/o mecanismos que nos permitan establecer de la mejor manera las prioridades que deben tenerse en cuenta en el análisis de las subpartidas que se incluirían en la negociación.

El presente estudio plantea dos abordajes como producto de la integración de los análisis de indicadores y el de matrices. El primero de ellos resulta de la construcción de una matriz de clasificación, mientras que el segundo se enfoca en la elaboración de una matriz de potencialidades. Esta última, a su vez, presenta dos alternativas: realizar un ordenamiento según los mercados de exportación más importantes para los productos peruanos o hacer una clasificación previa de aquellas subpartidas que presenten indicadores de VCR y DCR mayores de 1.

Los resultados encontrados en la matriz de clasificación indican que el orden de prioridad de los cuadrantes debe ser del primero al cuarto, es decir, de derecha a izquierda y de arriba a abajo. El primer cuadrante es el más importante, porque contiene las subpartidas en las que el Perú está aprovechando su capacidad de exportación hacia China. Por su parte, el segundo cuadrante representa las oportunidades que el Perú está desaprovechando, por lo que un análisis más completo hallaría los motivos por los que dichas subpartidas crecen por debajo del promedio ${ }^{16}$ y per- mitiría hallar mecanismos de solución para dirigir la fuerza exportadora del Perú a cubrir la demanda creciente del mercado chino. El tercer cuadrante, al presentar las subpartidas donde tanto la fuerza exportadora peruana como la demanda china decrecen, revela sectores claramente menos importantes en un contexto de negociación. Finalmente, el cuarto cuadrante incluye subpartidas con un mercado chino poco seguro en el futuro, lo que podría significar que los recursos empleados en impulsar el crecimiento de dichas exportaciones se podrían convertir en pérdidas.

En otras palabras, la priorización de las subpartidas en un contexto de negociación debe realizarse, en primer lugar, según la importancia de los cuadrantes componentes de cada matriz. Así, el ordenamiento comienza del primer al cuarto cuadrante. No obstante, el análisis debe complementarse con un estudio del tamaño de los mercados tanto ofertantes como demandantes, que puede ser el efectivo (matriz de clasificación) o el potencial (matriz de potencialidades). De este modo, la presente investigación hará posible también la priorización de las subpartidas dentro de cada cuadrante componente de la matriz, según la relevancia del monto del comercio bilateral actual o del que podría alcanzarse (potencial) de eliminarse los obstáculos. Finalmente, el último factor que permite mejorar o afinar el criterio de priorización es la clasificación de las subpartidas que obtengan de manera si-

16. Por ejemplo, habría que evaluar la producción nacional de esas subpartidas para determinar si la tasa de crecimiento por debajo del promedio se debe a una menor producción nacional y/o a su reorientación hacia el mercado interno. 
multánea el mayor número de índices superiores a 1. Lo anterior permite determinar cuáles son las subpartidas en las cuales se obtendría una mayor «eficiencia» tanto en la definición de los bienes que se deben exportar como en la de definición de los países adonde deben ser exportados. Así se asegura una mejor asignación de los recursos, a la vez que se evita la desviación del comercio.
Con el propósito de ampliar los alcances del presente estudio, un análisis posterior de los flujos comerciales deberá expandirse e incorporar los temas de aranceles y obstáculos técnicos al comercio. Asimismo, deberá aplicar la misma metodología para las exportaciones chinas hacia el Perú y realizar un examen comparativo con los resultados obtenidos en esta investigación. 


\section{Referencias bibliográficas}

APEC, Cooperación Económica del Asia Pacífico. 2004. APEC Economic Outlook. Secretaría General, Singapur.

ARÁOZ, M. y CASAS, Carlos. 1999. Revisión de las políticas de inversión en el Perú. Lima: Centro de Investigación de la Universidad del Pacífico.

BALASSA, BELA. 1965. Trade liberalization and revealed comparative advantages. Manchester School of Economics and Social Studies. Vol. 33, nº. 2, págs. 99-124.

CÓMEXPERÚ (Sociedad de Comercio Exterior del Perú). 2005. Perú exportó 37\% más en el 2004: EE.UU. y China impulsaron las exportaciones en toda Latinoamérica. $\mathrm{Se}$ manario Cómex n. ${ }^{\circ} 312$. Enero.

DELAGE, Fernando. 2004. China y el futuro de Asia.Política Exterior. Vol. 18, n. ${ }^{\circ} 102$, págs. 153-166.

DIRECÓN (Dirección General de Relaciones Económicas Internacionales de Chile). Informe de las conclusiones del estudio conjunto de factibilidad de un tratado de libre comercio entre Chile y China [www.direcon.cl].

ESTEVADERODAL, Antoni. 2005. El surgimiento de China: oportunidades y desafíos para América Latina y el Caribe. Presentación realizada en el seminario conmemo- rativo de los 40 Años de creación del Instituto para la Integración de América Latina y el Caribe (Intal). Buenos Aires, Argentina.

GUARDIA, Alexis. 2004. China en la globalización. Colección Ideal . Año 5, n. ${ }^{\circ} 46$.

HUANG, Y. 2000. China and the future of international trading system. En: Drysdale, Peter y Song, Lingang (eds.). China's entry to the WTO: Strategic issues and quantitative assessments. Londres: Routelegde Publishing.

IAP Study Group. 1997. APEC's progress toward the Bogor target: A quantitative assessment of individual plans. Tokio: PECC Japan Committee.

OMC (Organización Mundial del Comercio). 2004. Estadísticas del comercio internacional 2004. OMC.

TREJOS, Alberto. 2005. Desafíos y oportunidades para América Latina de la Expansión China. Presentación realizada en el Seminario conmemorativo de los 40 años de creación del Instituto para la Integración de América Latina y el Caribe (Intal), Buenos Aires, Argentina.

UNCTAD/WTO International Trade Centre (ITC). 1999. Tools for designing and monitoring national export strategies. Ginebra. 


\section{Anexo: Herramientas de comercio internacional}

\section{Índice de Ventajas Comparativas Reveladas (VCR)}

El Índice de VCR (Balassa, 1965) analiza las subpartidas exportadas y determina cuáles podrían presentar ventajas comparativas, las que para efecto del análisis actúan como una aproximación de la eficiencia del producto exportado.

Este índice se expresa de la siguiente manera:

$$
\left.V C R_{i h}=\left[\left(X_{i h} / X_{i}\right) / W_{w h} / W_{w h}\right)\right]
$$

Donde,

$X_{i h}=$ Exportaciones peruanas de la subpartida h.

$X_{i}=$ Exportaciones peruanas totales.

$W_{w h}=$ Comercio mundial de la subpartida $\mathrm{h}$.

$W_{w}=$ Comercio mundial total.

Como se puede observar, este índice compara la participación de una subpartida en el total de las exportaciones de un país con la participación de esa misma subpartida en el comercio mundial. De esta forma, el Perú presentará ventajas comparativas en las subpartidas cuyo índice de VCR sea mayor de 1 .

\section{Índice de Desventajas Comparativas Reveladas (DCR)}

El índice de DCR permite identificar a las subpartidas en las cuales China presenta una posición desventajosa en el comercio mundial. Este índice se calcula de la siguiente manera:

$$
\left.D C R_{j h}=\left[\left(M_{j h} / M_{j}\right) / W_{w h} / W_{w}\right)\right]
$$

Donde,

$M_{j h}=$ Importaciones chinas de la subpartida h.

$M_{j}=$ Importaciones chinas totales.

$W_{w h}=$ Comercio mundial de la subpartida h.

$W_{w}=$ Comercio mundial total.

Como se puede inferir, el ratio del numerador representa la participación del bien $h$ en las importaciones chinas, mientras el denominador indica la contribución del bien $h$ en el comercio mundial. De manera análoga al índice VCR, un resultado mayor de 1 significará que China posee desventajas comparativas en el ámbito mundial para la subpartida $h$.

\section{Índice de Complementariedad (IC)}

Teniendo en cuenta los resultados encontrados con los índices de VCR y DCR, el IC analiza, a partir de las estructuras comerciales, en qué medida dos economías, en este caso la peruana y la china, son compatibles. Este índice se construye de la siguiente manera:

$$
C_{i j}=\sum_{h}\left[\left(V C R_{i h}\right) \times\left(D C R_{j h}\right) \times\left(W_{w h} / W_{w}\right)\right]
$$


Donde,

$V C R_{i h}=$ Índice de Ventajas Comparativas Reveladas del Perú para h.

$D C R_{j h}=$ Índice de Desventajas Comparativas Reveladas de China para h.

$W_{w h}=$ Comercio mundial de la subpartida h.

$W_{w} \quad=$ Comercio mundial total.

De esta forma, un valor superior a 1 revela que la estructura exportadora peruana se complementa con la estructura importadora china. Este resultado podría interpretarse como una aproximación de una «menor» rivalidad comercial entre los países.

\section{Índice de Especialización (IE)}

El IE, como su nombre lo indica, busca identificar aquellos mercados en los que el Perú presenta algún nivel de especialización. Su fórmula se deriva de comparar la participación del Perú en las importaciones chinas de cierto producto con la participación de las exportaciones peruanas en el total de las importaciones chinas. Así:

$$
I E_{i j h}=\left[\left(X_{i j h} / M_{j h}\right) /\left(X_{i j} / M_{j}\right)\right]
$$

Donde,

$X_{i j h}=$ Exportaciones peruanas a China de la subpartida $\mathrm{h}$.

$M_{j h}=$ Importaciones chinas de la subpartida h.

$X_{i j}=$ Exportaciones totales del Perú a China.

$M_{j} \quad=$ Importaciones chinas totales.

Si el índice de especialización resulta mayor de 1, se puede concluir que el Perú se encuentra especializado en el mercado chino para la subpartida $h$.

\section{Cuota de Importaciones ( $\mathrm{CM}$ )}

El cálculo de la cuota de las importaciones chinas sobre el comercio global se realiza de la siguiente manera:

$$
C M_{j h}=\left[M_{j h} / W_{w h}\right]
$$

Donde,

$$
\begin{aligned}
M_{j h}= & \text { Importaciones chinas de la } \\
& \text { subpartida } \mathrm{h} . \\
W_{w h}= & \text { Comercio mundial de la } \\
& \text { subpartida } \mathrm{h} .
\end{aligned}
$$

Mientras más cerca de 1 se encuentre el resultado, mayor será la relevancia del mercado chino para las exportaciones mundiales de la subpartida $h$. 